\title{
On the Renormalization Group in Curved Spacetime
}

\author{
Stefan Hollands* and Robert M. Wald ${ }^{\dagger}$ \\ Enrico Fermi Institute, Department of Physics, \\ University of Chicago, 5640 Ellis Ave., \\ Chicago IL 60637, USA
}

September 10, 2018

\begin{abstract}
We define the renormalization group flow for a renormalizable interacting quantum field in curved spacetime via its behavior under scaling of the spacetime metric, $\mathbf{g} \rightarrow \lambda^{2} \mathbf{g}$. We consider explicitly the case of a scalar field, $\varphi$, with a self-interaction of the form $\kappa \varphi^{4}$, although our results should generalize straightforwardly to other renormalizable theories. We construct the interacting field - as well as its Wick powers and their time-ordered-products - as formal power series in the algebra generated by the Wick powers and time-ordered-products of the free field, and we determine the changes in the interacting field observables resulting from changes in the renormalization prescription. Our main result is the proof that, for any fixed renormalization prescription, the interacting field algebra for the spacetime $\left(M, \lambda^{2} \mathbf{g}\right)$ with coupling parameters $p$ is isomorphic to the interacting field algebra for the spacetime $(M, \mathbf{g})$ but with different values, $p(\lambda)$, of the coupling parameters. The map $p \rightarrow p(\lambda)$ yields the renormalization group flow. The notion of essential and inessential coupling parameters is defined, and we define the notion of a fixed point as a point, $p$, in the parameter space for which there is no change in essential parameters under renormalization group flow.
\end{abstract}

*Electronic mail: stefan@bert.uchicago.edu

$\dagger$ Electronic mail: rmwa@midway.uchicago.edu 


\section{Introduction}

Theories of a classical field in Minkowski spacetime that are derived from an action principle will automatically possess an invariance under a scaling of the global inertial coordinates of spacetime (or, equivalently, under scaling of the field momenta) provided that a corresponding scaling of the field amplitude and coupling constants are also performed in such a way that the action remains unchanged. If the quantum theory of this field is renormalizable, it turns out that in perturbation theory there also is a similar invariance of quantities of interest - such as the Green's functions of the fields - under scaling of the field momenta, but the required scaling of the field amplitudes and coupling constants differs, in general, from the simple scaling laws for the classical theory. This change of the "field strength normalization" and coupling constants under scaling is called the "renormalization group flow" of the theory. Important qualitative as well as quantitative information about quantum field theories can be gained from an analysis of their renormalization group flow.

For quantum field theories in Minkowski spacetime, there exist well known procedures for calculating the renormalization group flow in perturbation theory. In many cases, the picture obtained from low orders is believed to be at least in qualitative agreement with the behavior that would hold in the full, nonperturbatively constructed quantum field theory. Consequently, perturbative calculations of the renormalization group flow have played an important role in arguments concerning fundamental properties of quantum field theories. In particular, they form the basis of the claim that certain non-abelian gauge theories are "asymptotically free", i.e., that the gauge coupling flows towards zero at small distances (large momenta).

It is therefore of interest to know whether a similar scaling analysis can also be performed for perturbative interacting quantum field theory on an arbitrary globally hyperbolic curved (Lorentzian) spacetime. As we shall briefly review in section 2 below, the construction of perturbative interacting quantum field theory in curved spacetime has recently been achieved in [14], [15], based upon some earlier key results established in [3, 4] and other references. However, for at least the following two reasons, it does not seem possible to give a straightforward generalization to curved spacetime of the usual scaling analyses given for Minkowski spacetime. First, as already indicated above, the renormalization group flow in Minkowski spacetime is usually formulated in terms of behavior under the scaling of global inertial coordinates or, equivalently, scaling of the field momenta. However, in curved spacetime a formulation in terms of scaling of coordinates (or momenta) would introduce a very awkward and undesired coordinate dependence into the constructions. Also, since the scaling of coordinates no longer corresponds to a conformal isometry of the spacetime metric, one would not expect a simple behavior to occur under scalings of any coordinates. Second, the quantities whose scaling behavior is usually considered in studying the renormalization group flow in Minkowski space are the Green's functions of the interacting field or other quantities from which these can be derived, such 
as the "effective action". However, the Green's functions depend on a choice of state. For quantum field theories in Minkowski spacetime, this state would naturally be chosen to be the (unique) Poincare invariant vacuum state. However, even for a free quantum field in a general curved spacetime, there is no "preferred vacuum state" nor any other state that can be singled out for special consideration. Thus, even if a renormalization group flow could be defined in terms of Green's functions, there is no reason to expect it to be independent of the choice of state used to define the Green's functions.

A solution to the second difficulty is achieved by formulating the theory via the algebraic approach. In this approach, one views the observables as forming an abstract algebra, and one views the quantum states as suitable linear functionals on this algebra. This algebra is referred to as "abstract", because no representation of this algebra on a particular Hilbert space has been chosen from the outset, so that the (potentially problematic) issue of choosing states is completely disentagled from the issue of constructing the observables of the theory. As we shall see, the renormalization group flow can then be defined at the level of the algebra of observables.

The first difficulty above is solved by defining the renormalization group flow in terms of the behavior of the algebra of the interacting field under a scaling of the spacetime metric, $\mathbf{g} \rightarrow \lambda^{2} \mathbf{g}$, as has previously been suggested by other authors [21, 18, 19]. In Minkowski spacetime, the diffeomorphism defined by the rescaling of the global inertial coordinates, $x^{\mu} \rightarrow \lambda x^{\mu}$, is a conformal isometry with constant conformal factor $\lambda$, so rescaling the coordinates or momenta is equivalent to rescaling the spacetime metric. However, in a general curved spacetime there will not exist any conformal isometries, so rescaling the metric is not equivalent to any rescaling of coordinates or momenta. As we shall see, in perturbation theory the interacting field has a well defined behavior under scaling of the spacetime metric.

The results we shall obtain in this paper are based primarily on our previous uniqueness theorems [14 for Wick polynomials and their time-ordered products for a free quantum field. As we shall explain further in section 2 below, these results imply that the interacting field algebra is well defined up to certain renormalization ambiguities. In particular, for the case of a renormalizable theory, the ambiguities in the interacting field algebra correspond precisely to changes in the (finite number of) parameters appearing in the interaction Lagrangian'. This observation gives rise to the following means to define

\footnotetext{
${ }^{1}$ In other words, if one changes the prescription for defining Wick products and their time ordered products for the free theory in a manner compatible with the axioms of [14] and [15], the new interacting field algebra one obtains via the construction given in section 3 below will correspond to the interacting field algebra obtained with the original prescription, but with the interaction Lagrangian modified by the addition of terms of the same form as appearing in the original Lagrangian. The definition of the interacting field with the new prescription will also correspond up to a numerical factor to the definition of the interacting field in the corresponding algebra obtained from the original prescription with the modified Lagrangian, i.e., the isomorphism of the interacting field algebras for the two different prescriptions will map the interacting field to a multiple of the interacting field. It should be noted, however, that the new definition of higher Wick powers of the interacting field (as well as time-ordered-products of Wick
} 
the renormalization group flow: Fix a renormalization prescription for defining the free field Wick polynomials and their time ordered products. Now apply this renormalization prescription to define Wick polynomials and their time-ordered-products for free quantum fields on the spacetime $\left(M, \lambda^{2} \mathbf{g}\right)$, with all of the parameters of the theory also scaled according to their "engineering dimension" (i.e., scaled in such a way as to keep the classical action invariant). The free field algebra of observables $\mathcal{W}(M, \mathbf{g})$ (defined in [14] and in section 2 below) is naturally isomorphic to $\mathcal{W}\left(M, \lambda^{2} \mathbf{g}\right)$ with scaled parameters, and we can use this isomorphism to define a new ( $\lambda$-dependent) renormalization prescription for Wick polynomials and their time-ordered products on the original spacetime $(M, \mathbf{g})$. We thereby obtain a new ( $\lambda$-dependent) prescription for defining the interacting field algebra. However, by our uniqueness results, this prescription must be equivalent to the original prescription for defining the interacting field algebra modulo a change of parameters appearing in the interaction Lagrangian. Consequently, we get a $\lambda$-dependent "flow" in the parameter space of the interacting theory"2. This flow defines the action of the renormalization group for a quantum field in curved spacetime.

In order to implement the above ideas, we first must define the interacting quantum field algebra and therefore must address the following two difficulties: (i) As in Minkowski spacetime, the interacting quantum field is defined only perturbatively, and it is not expected that the perturbation series converges. (ii) The usual formula for defining the interacting field expresses it in terms of a free "in"-field [12]. Even if the theory under consideration is such that in Minkowski spacetime the interacting field approaches a free "in"-field in the asymptotic past in a suitable sense, there is no reason to expect any such behavior to occur in an arbitrary globally hyperbolic curved spacetime.

As discussed in section 3.1, we shall, in essence, sidestep issue (i) by treating the interacting field algebra only at the level of a formal perturbation series. In other words, we do not attempt to define the interacting field algebra at a finite value of a nonlinear coupling parameter, $\kappa$, but simply consider the algebra generated by the formal perturbation series expressions in $\kappa$. In this respect, our analysis is neither better nor worse than the corresponding analyses for perturbative quantum field theory in Minkowski spacetime. We note, however, that at least some of the difficulties encountered in making sense of perturbative expansions for nonlinear quantum field theory may be due to the non-analytic

powers of the interacting field) will not correspond to the definition of these quantities obtained from the original prescription with the modified Lagrangian. Instead, under the isomorphism of the algebras, a higher Wick power (or a time-ordered-product of Wick powers) will, in general, be mapped into a field of the form specified in eq. (52) below.

${ }^{2}$ In other words, if we scale the spacetime metric and correspondingly scale the parameters, $p_{0}$, of the free Lagrangian, $\boldsymbol{L}_{0}$, according to their "engineering dimension", then the resulting theory is equivalent to a theory where the metric and parameters, $p_{0}$, are not scaled, but the interaction Lagrangian, $\boldsymbol{L}_{1}$, is modified by $\lambda$-dependent terms of the same form as appear in the (full) Lagrangian $\boldsymbol{L}=\boldsymbol{L}_{0}+\boldsymbol{L}_{1}$. It should be emphasized that it is far from obvious that, for a perturbatively constructed interacting theory, a change in a parameter appearing in $\boldsymbol{L}_{1}$ as occurs in the renormalization group flow is equivalent to a corresponding change in that parameter in $\boldsymbol{L}_{0}$; see the end of section 4.1 for further discussion. 
behavior of ground states and/or "in" and "out" states. It appears conceivable that at least some of the difficulties of perturbation theory could be averted if one works strictly at the algebraic level and uses perturbation formulas only to obtain algebraic relationships between interacting field observables (thereby defining the interacting field algebra) rather than using perturbation theory to calculate quantities involving, say, ground states or "in" and "out" states. However, we shall not attempt to pursue these ideas in this paper.

On the other hand, difficulty (ii) can be genuinely overcome by properly taking limits as the cutoff on the interaction is removed: The Bogoliubov formula defining the interacting field (see eq. (35) below) is well defined if the nonlinear coupling parameter, $\kappa$, is taken to be a smooth function of compact support, so that the nonlinear interaction is "turned off" in the past and future. If one then attempts to take a limit where $\kappa$ approaches a constant, difficulties may arise if one demands that the interacting field remain fixed in, say, the asymptotic past. However, no difficulties arise if, following the ideas of [4], we demand that the interacting field remain fixed in the "interior" of the spacetime as $\kappa$ approaches a constant. This construction is given in section 3.1.

The organization of this paper is as follows. In section 2 , we briefly review the main ingredients that we will need from free quantum field theory in curved spacetime, including the definition and uniqueness properties of Wick powers and their time-ordered-products. In section 3.1 we give the construction of the interacting field and in section 3.2 we characterize its renormalization ambiguities. The scaling behavior of the interacting theory is analyzed in section 4.1, and the renormalization group flow is defined. The notions of essential and inessential coupling parameters and the notion of "fixed points" under the renormalization group flow are defined in section 4.2. In appendix B, we will relate our rather abstract formulation of renormalization theory and the renormalization group flow at the algebraic level to more usual formulations in terms of Feynman diagrams.

In this paper, we will consider only a scalar field with Lagrangian density of the form

$$
\boldsymbol{L}=\boldsymbol{L}_{0}+\boldsymbol{L}_{1} \equiv \frac{1}{2}\left[(\nabla \varphi)^{2}+m^{2} \varphi^{2}+\xi R \varphi^{2}+\kappa \varphi^{4}\right] \boldsymbol{\epsilon},
$$

where, $R$ is the scalar curvature and $\boldsymbol{\epsilon}$ is the volume element constructed from the spacetime metric $\mathbf{g}=\mathbf{g}_{a b}$. The self-interaction $\boldsymbol{L}_{1}=\frac{1}{2} \kappa \varphi^{4} \boldsymbol{\epsilon}$ will be treated perturbatively. However, all of our analysis should generalize straightforwardly to other renormalizable quantum field theories.

Our notation and conventions follow those of our previous papers [14], [15]. All spacetimes $(M, \mathbf{g})$ considered in this paper will be assumed to be globally hyperbolic and time oriented. We will denote the free quantum scalar field (defined by the Lagrangian (1) with $\kappa=0)$ by $\varphi$ and will use the generic notation $\Phi$ to denote other local covariant fields in the free theory. The interacting field will be denoted $\varphi_{L_{1}}$ and other local covariant fields in the interacting theory will be denoted $\Phi_{L_{1}}$. In this paper, all fields will be smeared with scalar densities (of unit weight); we will denote the space of smooth unit weight scalar 
densities of compact support on $M$ by $\mathcal{D}_{1}(M)$.

\section{The free quantum field in curved spacetime}

The perturbative construction of a self-interacting quantum scalar field in curved spacetime is based upon the construction of the free quantum field theory. In this section, we consider the quantum field theory of a free scalar field $\varphi$, described by the classical Lagrangian density

$$
\boldsymbol{L}_{0}=\frac{1}{2}\left[(\nabla \varphi)^{2}+m^{2} \varphi^{2}+\xi R \varphi^{2}\right] \boldsymbol{\epsilon} .
$$

Note that under a scaling of metric, $\mathbf{g} \rightarrow \lambda^{2} \mathbf{g}$ with $\lambda$ a positive constant, the Lagrangian density remains invariant provided that we also scale the field, $\varphi$, mass, $m$, and coupling parameter $\xi$, by $\varphi \rightarrow \lambda^{-1} \varphi, m \rightarrow \lambda^{-1} m, \xi \rightarrow \xi$. We refer to the power of $\lambda$ appearing in these scaling rules as the engineering dimension of the quantity. More generally, any monomial, $\Phi$, constructed out of $\varphi$ and its derivatives, the curvature, and the coupling constants $m$ and $\xi$ will have a well defined engineering dimension, denoted $d_{\Phi}$.

As is well known, in a general curved spacetime, there is no "preferred vacuum state" nor even any preferred Hilbert space construction of the quantum theory corresponding to the classical Lagrangian (2) (see, e.g., [22] for further discussion). Therefore, in our view, it is essential to formulate the theory via the algebraic approach.

As in [14, we shall take the algebra of observables of the free field to be the "extended Wick polynomial algebra" $\mathcal{W}(M, \mathbf{g})$. As described in [14, this algebra can be constructed by choosing a quasifree Hadamard state, $\omega$, on the "canonical commutation algebra", $\mathcal{A}(M, \mathbf{g})$, then considering the normal ordered field operators on the GNS representation of $\omega$, and showing [3] that one gets well defined operators by smearing these normal ordered operators with suitable distributions rather than test functions. The resulting algebra of operators can then be shown [14] to be independent of the choice of $\omega$.

Following [8], we outline here a much more direct construction of $\mathcal{W}(M, \mathbf{g})$. This construction is sufficiently different in appearance from that given in [14] that it is worthwhile to explain the relationship between the constructions. First, recall the usual construction of the canonical commutation algebra, $\mathcal{A}(M, \mathbf{g})$ : Start with the free *-algebra generated by the identity, $\mathbb{1}$, and all expressions of the form $\varphi(f)$, where $f$ is an element of $\mathcal{D}_{1}(M)$, the space of smooth scalar densities on $M$ with compact support. (Thus, this algebra consists of all finite linear combinations of $\mathbb{1}$ and terms containing finitely many factors of the form $\varphi\left(f_{i}\right)$ and $\varphi\left(f_{j}\right)^{*}$.) Next, define the two-sided ideal consisting of all elements of this algebra that contain at least one factor of any of the following four types:

(i) $\varphi\left(\alpha_{1} f_{1}+\alpha_{2} f_{2}\right)-\alpha_{1} \varphi\left(f_{1}\right)-\alpha_{2} \varphi\left(f_{2}\right)$, with $\alpha_{1}, \alpha_{2} \in \mathbb{C}$;

(ii) $\varphi(f)^{*}-\varphi(\bar{f})$; 
(iii) $\varphi\left(\left(\nabla^{a} \nabla_{a}-m^{2}-\xi R\right) f\right)$; and

(iv) $\varphi\left(f_{1}\right) \varphi\left(f_{2}\right)-\varphi\left(f_{2}\right) \varphi\left(f_{1}\right)-i \Delta\left(f_{1}, f_{2}\right) \mathbb{1}$, where $\Delta$ denotes the advanced minus retarded Green's function for the Klein-Gordon operator.

Then $\mathcal{A}(M, \mathbf{g})$ is defined by factoring the free algebra by this ideal.

It is useful to make the following trivial change in the construction of $\mathcal{A}(M, \mathbf{g})$ : Instead of starting with the free algebra generated by the identity, $\mathbb{1}$, and symbols of the form $\varphi(f)$, we start with the free tensor algebra of smooth compactly supported scalar test densities on $M$,

$$
\mathcal{F}(M) \equiv \mathbb{C} \oplus \bigoplus_{n \geq 1} \otimes^{n} \mathcal{D}_{1}(M)
$$

with a ${ }^{*}$-operation defined by complex conjugation. (Note that although the direct sum in eq. (3) is infinite, by definition, each element of $\mathcal{F}(M)$ has only finitely many non-zero entries.) The ${ }^{*}$-algebra $\mathcal{F}(M)$ already incorporates the identifications corresponding to (i) and (ii) above, and clearly is isomorphic to the free algebra of the previous paragraph factored by the ideal generated by (i) and (ii). Thus, we can equivalently define $\mathcal{A}(M, \mathbf{g})$ by factoring $\mathcal{F}(M)$ by the ideal generated by expressions (iii) and (iv) above. We will incorporate this viewpoint in our notation by denoting elements of $\mathcal{A}(M, \mathbf{g})$ by their representatives in $\mathcal{F}(M)$. Thus, for example, we will denote the element of $\mathcal{A}(M, \mathbf{g})$ corresponding to the field operator smeared with $f \in \mathcal{D}_{1}(M)$ by $[f]$ rather than $\varphi(f)$.

Next, we note that given any $t \in \mathcal{F}(M)$, the imposition of the commutation relations (iv) above would allow us to choose a unique representative of $t$ in the totally symmetric tensor algebra. Thus, rather than imposing these commutation relations by factorization as above, we may instead work with the totally symmetric tensor algebra. Hence, we define

$$
\mathcal{F}_{\text {sym }}(M) \equiv \mathbb{C} \oplus \bigoplus_{n \geq 1} \otimes_{\text {sym }}^{n} \mathcal{D}_{1}(M)
$$

and we define a product, $\star_{0}$, (which depends upon $\mathbf{g}$ ) in $\mathcal{F}_{\text {sym }}(M)$ that corresponds to taking the ordinary tensor product in $\mathcal{F}(M)$. Namely, if $t_{n} \in \otimes_{\text {sym }}^{n} \mathcal{D}_{1}(M)$ and $s_{m} \in$ $\otimes_{\text {sym }}^{m} \mathcal{D}_{1}(M)$, we define

$$
\begin{array}{r}
\left(t_{n} \star_{0} s_{m}\right)_{n+m-2 k}\left(x_{1}, \ldots, x_{n+m-2 k}\right)=\frac{n ! m !}{k !(n-k) !(m-k) !} \mathbb{S} \int_{M^{2 k}} t_{n}\left(y_{1}, \ldots, y_{k}, x_{1}, \ldots, x_{n-k}\right) \\
s_{m}\left(y_{k+1}, \ldots, y_{2 k}, x_{n-k+1}, \ldots, x_{n+m-2 k}\right) \prod_{i=1}^{k} \frac{i}{2} \Delta\left(y_{i}, y_{k+i}\right), \quad \text { (5) }
\end{array}
$$

where "S" denotes total symmetrization in the variables $x_{1}, \ldots, x_{n+m-2 k}$ and where the integral is over the " $y$ "-variables?. In other words, the right side of eq. (5) gives the

\footnotetext{
${ }^{3}$ Since $t_{n}$ and $s_{m}$ are densities, no volume element has to be specified in the integral.
} 
totally symmetric representative of $t_{n} \otimes s_{m}$ in the tensor algebra $\mathcal{F}(M)$ under imposition of the commutation relations (iv). Since the algebra (4) with the product (5) already incorporates conditions (i), (ii), and (iv) above, we consider the ideal consisting of all elements of $\mathcal{F}_{\text {sym }}(M)$ that contain at least one factor of the form $\left(\nabla^{a} \nabla_{a}-m^{2}-\xi R\right) f$. We again obtain $\mathcal{A}(M, \mathbf{g})$ by factoring $\mathcal{F}_{\text {sym }}(M)$ by this ideal.

We now make an important further modification to the above construction by introducing a new ( $\omega$-dependent) product, $\star$, on $\mathcal{F}_{\text {sym }}(M)$ by replacing $\frac{i}{2} \Delta$ in eq. (5) by $\omega$ where $\omega$ is an arbitrary ("undensitized") distribution in two variables that satisfies the Klein-Gordon equation in each variable and whose antisymmetric part is equal to $\frac{i}{2} \Delta$,

$$
\begin{array}{r}
\left(t_{n} \star s_{m}\right)_{n+m-2 k}\left(x_{1}, \ldots, x_{n+m-2 k}\right)=\frac{n ! m !}{k !(n-k) !(m-k) !} \mathbb{S} \int_{M^{2 k}} t_{n}\left(y_{1}, \ldots, y_{k}, x_{1}, \ldots, x_{n-k}\right) \\
s_{m}\left(y_{k+1}, \ldots, y_{2 k}, x_{n-k+1}, \ldots, x_{n+m-2 k}\right) \prod_{i=1}^{k} \omega\left(y_{i}, y_{k+i}\right), \quad \text { (6) }
\end{array}
$$

where the integral is again over the " $y$ "-variables. Then, by the same argument as in Lemma 2.1 of [14, it can be seen that $\mathcal{F}_{\text {sym }}(M)$ with the product $\star$ is naturally isomorphic to $\mathcal{F}_{\text {sym }}(M)$ with the product $\star_{0}$. Therefore if we factor $\mathcal{F}_{\text {sym }}(M)$ with the product $\star$ by the ideal comprised by all elements of $\mathcal{F}_{\text {sym }}(M)$ that contain at least one factor of $\left(\nabla^{a} \nabla_{a}-m^{2}-\xi R\right) f$, we again obtain an algebra isomorphic to $\mathcal{A}(M, \mathbf{g})$. It also should be noted that for $f_{1}, f_{2} \in \mathcal{D}_{1}(M)$ we have

$$
f_{1} \star f_{2}-f_{2} \star f_{1}=i \Delta\left(f_{1}, f_{2}\right) \mathbb{1} .
$$

Now, choose $\omega$ to be the two-point function of a Hadamard state. Then the product (6) corresponds to Wick's formula expressing the product of a normal-ordered $n$-point function with a normal ordered $m$-point function in terms of normal ordered products, where the normal ordering is done with respect to the quasi-free Hadamard state with two-point function $\omega$. It can thereby be seen that for any $t_{n} \in \otimes_{\mathrm{sym}}^{n} \mathcal{D}_{1}(M)$ of the form $t_{n}=f_{1} \otimes_{\text {sym }} \cdots \otimes_{\text {sym }} f_{n}$ with each $f_{i} \in \mathcal{D}_{1}(M)$, the algebraic element $\left[t_{n}\right] \in \mathcal{A}(M, \mathbf{g})$ corresponding to the equivalence class of $t_{n}$ is represented by the normal ordered product $: \varphi\left(f_{1}\right) \cdots \varphi\left(f_{n}\right):_{\omega}$ in the GNS-representation of the state $\omega$.

The key observation needed to define the algebra $\mathcal{W}(M, \mathbf{g})$ is to note that the wavefront set properties of $\omega$ then imply that eq. (6) continues to make sense when the test function space $\otimes_{\text {sym }}^{n} \mathcal{D}_{1}(M)$ in (4) is replaced by the much larger space

$\mathcal{E}_{\text {sym }}^{\prime}\left(M^{\times n}\right)=\left\{\right.$ compactly supp. symm. distr. $\left.t_{n} \mid \mathrm{WF}\left(t_{n}\right) \subset T^{*} M^{n} \backslash\left(V_{+}^{\times n} \cup V_{-}^{\times n}\right)\right\}$,

${ }^{4}$ Since the elements in $\mathcal{E}_{\text {sym }}^{\prime}\left(M^{\times n}\right)$ are distributions, they automatically have the character of densities. The space $\otimes_{\text {sym }}^{n} \mathcal{D}_{1}(M)$ can therefore be naturally identified with a subspace of $\mathcal{E}_{\text {sym }}^{\prime}\left(M^{\times n}\right)$, without the need to specify a volume element on $M$. 
where $V_{ \pm}$is the future/past lightcone with respect to the metric $\mathbf{g}$, and where "WF" denotes the wave-front set of a distribution [13]. We define $\mathcal{W}(M, \mathbf{g})$ to be the vector space

$$
\mathcal{E}^{\prime}(M, \mathbf{g}) \equiv \mathbb{C} \oplus \bigoplus_{n \geq 1} \mathcal{E}_{\text {sym }}^{\prime}\left(M^{\times n}\right)
$$

with product (6), factored by the ideal comprised by all elements of the form $\left(\nabla^{a} \nabla_{a}-\right.$ $\left.m^{2}-\xi R\right)_{x_{i}} t_{n}\left(x_{1}, \ldots, x_{n}\right)$. Thus, every element $a \in \mathcal{W}$ corresponds to an equivalence class $a=[s]$ of an element $s=s_{0}+\sum_{k=1}^{n} s_{k}$, where $s_{0} \in \mathbb{C}$, and where $s_{k} \in \mathcal{E}_{\text {sym }}^{\prime}\left(M^{\times k}\right)$. The product of two elements in $\mathcal{W}$ is given by $[s] \star[t] \equiv[s \star t]$. If $f$ is a smooth scalar density on $M$ of compact support, then the equivalence class $[f] \in \mathcal{W}$ corresponds exactly to the smeared free field $\varphi(f)$.

The definition of the algebra $\mathcal{W}$ a priori depends on some choice for $\omega$, but it was shown in 14] that different choices for $\omega$ give rise to isomorphic algebras. Therefore, as an abstract algebra, $\mathcal{W}$ is independent of this choice. Since $\mathcal{A}$ is naturally a subalgebra of $\mathcal{W}$, we automatically know what elements of $\mathcal{W}$ correspond to the smeared field $\varphi(f)$ and its smeared $n$-point functions. However, it is not obvious what (if any) elements of $\mathcal{W}$ correspond to smeared Wick powers of the field and time-ordered products of Wick powers.

This issue was addressed in [14] and [15], where an axiomatic approach was taken. A key condition imposed in [14] and [15] on the definition of Wick powers and their timeordered-products was that they be local, covariant fields [5]. In order to define this notion, it is necessary to think of the fields as being defined not only for a given, fixed spacetime, but rather for all (globally hyperbolic) spacetimes, and we incorporate this viewpoint here by indexing the field with the spacetime under consideration, such as $\Phi[M, \mathbf{g}]$. If $(M, \mathbf{g})$ and $(\tilde{M}, \tilde{\mathbf{g}})$ are two spacetimes such that there is a causality preserving isometric embedding, $\chi$, of $(\tilde{M}, \tilde{\mathbf{g}})$ into $(M, \mathbf{g})$, then the algebra $\mathcal{W}(\tilde{M}, \tilde{\mathbf{g}})$ can be regarded as a subalgebra of $\mathcal{W}(M, \mathbf{g})$ via a homomorphism $\alpha_{\chi}$ in a natural way [14], so that the free field theory with algebra $\mathcal{W}(M, \mathbf{g})$ is a local, covariant field theory [5]. The requirement that $\Phi$ be a local covariant field is then that

$$
\alpha_{\chi}(\Phi[\tilde{M}, \tilde{\mathbf{g}}](x))=\Phi[M, \mathbf{g}](\chi(x)) .
$$

It was shown in [14] that this requirement together with a number of additional requirements (such as commutation properties, continuity and analyticity conditions, microlocal spectral conditions, and causal factorization) uniquely determines the definition of Wick powers and their time-ordered-product up to certain well defined renormalization ambiguities. Existence of Wick powers satisfying these properties also was established in [14], and existence of their time-ordered-products was proven in [15].

The results of the present paper will rely heavily on the uniqueness theorem 5.2 of [14] for time-ordered-products. The allowed ambiguity in the definition of time-orderedproducts as given in theorem 5.2 of [14] is rather awkward to state, so we find it useful 
to reformulate this theorem in the following manner (see [1, 8]). First, we introduce an abstract vector space, $\mathcal{V}$, comprised by finite linear combinations of basis elements labeled by formal products of $\varphi$ and its covariant derivatives,

$$
\mathcal{V}=\operatorname{span}_{\mathbb{C}}\left\{\Phi=\prod \nabla_{\left(a_{1}\right.} \cdots \nabla_{\left.a_{i}\right)} \varphi\right\}
$$

We refer to the elements of $\mathcal{V}$ as "formal" because we do not assume any relations between the fields at this stage. In particular, we regard the field and its derivatives as independent quantities which are not related by the field equation. Let

$$
\mathcal{D}_{1}(M, \mathcal{V}) \equiv\{\text { smooth densities on } M \text { of compact support with values in } \mathcal{V}\}
$$

so that an element $F \in \mathcal{D}_{1}(M, \mathcal{V})$ can be uniquely expressed as a finite sum $F=\sum f_{i} \Phi_{i}$ with each $\Phi_{i}$ a basis element of $\mathcal{V}$ and $f_{i} \in \mathcal{D}_{1}(M)$. It is convenient to think of a prescription for defining Wick powers as a linear map from $\mathcal{D}_{1}(M, \mathcal{V})$ into the algebra $\mathcal{W}(M, \mathbf{g})$. Thus, a prescription for Wick powers associates to an element $f(x) \Phi \in \mathcal{D}_{1}(M, \mathcal{V})$ an element $\Phi(f) \in \mathcal{W}(M, \mathbf{g})$. Similarly, it is useful to view the $n$-fold time ordered product of Wick powers as an $n$-times multilinear map

$$
\begin{aligned}
T: X^{n} \mathcal{D}_{1}(M, \mathcal{V}) & \rightarrow \mathcal{W}(M, \mathbf{g}) \\
\left(f_{1} \Phi_{1}, \ldots, f_{n} \Phi_{n}\right) & \rightarrow T\left(\prod \Phi_{i}\left(f_{i}\right)\right)
\end{aligned}
$$

The map defining Wick powers is, of course, the special case $n=1$ of the map defining time-ordered-products.

Let us now suppose that we have two prescriptions for defining time-ordered-products (and, in particular, two prescriptions for defining Wick powers). It is simplest and most convenient to express the formula for the difference between these prescriptions in terms of the local $S$-matrix, $S\left(\sum f_{i} \Phi_{i}\right)$, for the formal sum $\sum f_{i} \Phi_{i}$, which is formally defined by

$$
S\left(\sum f_{i} \Phi_{i}\right)=\mathbb{1}+\sum_{n \geq 1} \frac{i^{n}}{n !} T\left(\prod^{n} \sum \Phi_{i}\left(f_{i}\right)\right) .
$$

(Of course, as discussed further at the beginning of section 3.1 below, the series on the right side of eq. (15) is not expected to converge. It should be viewed as merely a bookeeping device that will allow us to write an infinite sequence of complicated equations - given explicitly in eq. (25) below - as a single equation.) Denote the image of the $n$-tuple $\left(f_{1} \Phi_{1}, \ldots, f_{n} \Phi_{n}\right) \in \times{ }^{n} \mathcal{D}_{1}(M, \mathcal{V})$ under the first prescription as $T\left(\prod \Phi_{i}\left(f_{i}\right)\right)$ and denote its image under the second prescription as $\tilde{T}\left(\prod \tilde{\Phi}_{i}\left(f_{i}\right)\right)$. Then, if both prescriptions satisfy all of the requirements stated in [14], [15], theorem 5.2 of [14] establishes that the following relation holds between the corresponding local $S$-matrices:

$$
\tilde{S}\left(\sum f_{i} \Phi_{i}\right)=S\left(\sum f_{i} \Phi_{i}+\delta\left(\sum f_{i} \Phi_{i}\right)\right)
$$


where $\delta\left(\sum f_{i} \Phi_{i}\right)$ is given by the formal power series expression

$$
\delta\left(\sum f_{i} \Phi_{i}\right)=\sum_{n \geq 1} \frac{i^{n-1}}{n !} O_{n}\left(\chi^{n} \sum f_{i} \Phi_{i}\right) .
$$

Equation (16) is to be interpreted as an infinite sequence of equalities between terms containing equal numbers of each of the $f_{i}$ 's under the formal substitutions (15) and (17). In eq. (17), the $O_{n}$ 's are multilinear maps

$$
O_{n}: \chi^{n} \mathcal{D}_{1}(M, \mathcal{V}) \rightarrow \mathcal{D}_{1}(M, \mathcal{V})
$$

of the form:

$$
O_{n}\left(\times_{i=1}^{n} f_{i} \Phi_{i}\right)=\sum_{j} F_{j} \Psi_{j}
$$

where $\Psi_{j}$ are basis fields in $\mathcal{V}$ and the densities $F_{j}$ are of the form

$$
F_{j}(x)=\boldsymbol{\epsilon}(x) \sum_{(a)=\left(a_{1}\right) \ldots\left(a_{n}\right)} C_{j}^{(a)}(x) \prod_{i=1}^{n} \nabla_{\left(a_{i}\right)} f_{i}(x) .
$$

In this formula, we have idenfied the densities $f_{i}$ with test functions on $M$ using the metric volume element $\epsilon$ and we have used the multi-index notation $\nabla_{(a)}=\nabla_{\left(a_{1}\right.} \cdots \nabla_{\left.a_{s}\right)}$. The quantities $C_{j}^{(a)}$ are tensors that are monomials in the Riemann tensor, its covariant derivatives, and $m^{2}$, with coefficients that are analytic functions of $\xi$. The quantities $O_{n}$ are further restricted by the requirement that

$$
\left[T\left(O_{n}\left(\times_{i=1}^{n} f_{i} \Phi_{i}\right)\right), \varphi\left(f_{n+1}\right)\right]=\sum_{k=1}^{n} T\left(O_{n}\left(f_{1} \Phi_{1}, \ldots, i \sum_{(a)}\left(f_{n+1} \Delta_{(a)} f_{k}\right) \frac{\partial \Phi_{k}}{\partial \nabla_{(a)} \varphi}, \ldots, f_{n} \Phi_{n}\right)\right) .
$$

Here, $\partial \Phi / \partial \nabla_{(a)} \varphi$ is the element in $\mathcal{V}$ obtained by formally differentiating the expression $\Phi \in \mathcal{V}$ with respect to $\nabla_{(a)} \varphi$ (thereby viewing the latter as an "independent variable"), (a) is a spacetime multi-index as above, and

$$
\left(f_{n+1} \Delta_{(a)} f_{i}\right)(x)=\int_{M} f_{n+1}(x) \Delta(x, y) \nabla_{(a)} f_{i}(y),
$$

where $\Delta$ is the advanced minus retarded Green's function, and where the integration is over the " $y$ "-variables. In addition, if $d_{j}{ }^{(a)}$ is the engineering dimension of $C_{j}^{(a)}, N_{(a)}$ the number of covariant derivatives appearing explicitly in equation (20), and $d_{j}$ is the engineering dimension of the field $\Psi_{j}$, then each of the terms in the sum (20) must satisfy the power counting relation

$$
\sum_{i=1}^{n} d_{\Phi_{i}}=4 n+N_{(a)}+d_{j}^{(a)}+d_{j}
$$


for all multi-indices $(a)$ and all $j$. Furthermore, the quantities $\delta(f \Phi)$ defined in eq. (17) satisfy the reality condition

$$
\delta(f \Phi)^{*}=\delta(f \Phi)
$$

for real valued $f$ and hermitian $\Phi$, which corresponds to the unitarity requirement, $S(f \Phi)^{-1}=S(f \Phi)^{*}$, for real valued $f$ and hermitian $\Phi$. Equation (24) is equivalent to the reality property $O_{n}\left(\times^{n} f \Phi\right)^{*}=(-1)^{n-1} O_{n}\left(\times^{n} f \Phi\right)$.

The relations between the two prescriptions for time-ordered-products given implicitly in eq. (16) can be written out explicitly as

$$
\tilde{T}\left(\prod_{i=1}^{n} \tilde{\Phi}_{i}\left(f_{i}\right)\right)=T\left(\prod_{i=1}^{n} \Phi_{i}\left(f_{i}\right)\right)+\sum_{P} T\left(\prod_{I \in P} O_{|I|}\left(\times_{j \in I} f_{j} \Phi_{j}\right) \prod_{i \notin I} \Phi_{\forall I \in P}\left(f_{i}\right)\right) .
$$

where, $P$ is a collection of pairwise disjoint subsets $I_{1}, I_{2}, \ldots$ of the set $\{1, \ldots, n\}$, not all of which can be empty, and $|I|$ is the number of elements of such a set. Equation (25) corresponds to our previous formulation of the uniqueness theorem given in theorem 5.2 of [14], except that, for simplicity, we asssumed in the statement of that theorem that the "untilded" prescription for defining Wick products was given by "local normal ordering" with respect to a local Hadamard parametrix. In Minkowski spacetime a proof that eq. (25) corresponds to the formal expansion of eq. (16) is given in [20, thm. 6.1]; the combinatorical arguments given there can be generalized in a straightforward manner to the present case.

If we take $\sum f_{i} \Phi_{i}$ to be the interaction Lagrangian density, then eq. (16) corresponds to the familiar statement in perturbative quantum field theory in Minkowski spacetime that the "renormalization ambiguities" in the $S$-matrix correspond simply to adding "counterterms" to the Lagrangian of the appropriate "power counting" dimension. The only significant difference occurring when one goes to curved spacetime is that additional counterterms involving the spacetime curvature may occur.

We conclude this section by reviewing the scaling properties of Wick powers and their time-ordered-products. Fix a Wick power $\Phi[M, \mathbf{g}, p]$ and consider the 1-parameter family of Wick powers $\Phi\left[M, \lambda^{2} \mathbf{g}, p(\lambda)\right]$ defined on the spacetimes $\left(M, \lambda^{2} \mathbf{g}\right)$, with coupling constants

$$
p(\lambda)=\left(\lambda^{-2} m^{2}, \xi\right)
$$

These quantities belong (when smeared with a test density) to different algebras,

$$
\Phi\left[M, \lambda^{2} \mathbf{g}, p(\lambda)\right](f) \in \mathcal{W}\left(M, \lambda^{2} \mathbf{g}, p(\lambda)\right)
$$

\footnotetext{
${ }^{5}$ We should emphasize that our interest here is not in determining the renormalization ambiguities in a global scattering matrix (which will, in general, not even be defined) but rather in determining the renormalization ambiguities in the interacting field itself (as well as its Wick powers and the time-orderedproducts of its Wick powers). However, the formulas expressing these ambiguities are most conveniently expressed in terms of the relative $S$-matrix, which is defined in terms of the local $S$-matrix (see section 3.2 below), so a knowledge of the ambiguities in the local $S$-matrix will enable us to determine the ambiguities in the interacting field.
} 
(where we now have indicated explicitly the dependence of this algebra and the field on the coupling parameters $p$ ), and hence cannot be compared directly. However, as observed in [14, one can define a natural *-isomorphism

$$
\sigma_{\lambda}: \mathcal{W}\left(M, \lambda^{2} \mathbf{g}, p(\lambda)\right) \rightarrow \mathcal{W}(M, \mathbf{g}, p), \quad \sigma_{\lambda}\left(\left[t_{n}\right]\right) \equiv \lambda^{-n}\left[t_{n}\right]
$$

In other words, $\sigma_{\lambda}$ maps the element of $\mathcal{W}\left(M, \lambda^{2} \mathbf{g}, p(\lambda)\right)$ corresponding to $: \varphi\left(f_{1}\right) \cdots \varphi\left(f_{n}\right):_{\omega_{\lambda}}$ in the GNS-representation of the quasi-free Hadamard state $\omega_{\lambda}$ into the element of $\mathcal{W}(M, \mathbf{g}, p)$ corresponding to $: \varphi\left(f_{1}\right) \cdots \varphi\left(f_{n}\right):_{\omega}$ in the GNS-representation of the quasi-free Hadamard state $\omega$, where the two-point functions of $\omega_{\lambda}$ and $\omega$ are related by $\omega_{\lambda}\left(x_{1}, x_{2}\right)=$ $\lambda^{-2} \omega\left(x_{1}, x_{2}\right)$. Using this isomorphism, we can then identify the Wick product $\Phi\left[M, \lambda^{2} \mathbf{g}, p(\lambda)\right]$ with a local covariant field $\sigma_{\lambda}\left(\Phi\left[M, \lambda^{2} \mathbf{g}, p(\lambda)\right]\right)$ for the unscaled metric and unscaled coupling constants $\mathbf{g}, p$.

The free field $\varphi$ has the homogeneous scaling behavior

$$
\sigma_{\lambda}(\varphi(f))=\lambda^{-1} \varphi(f)
$$

where the field on the left side of this equation is defined in terms of the scaled metric $\lambda^{2} \mathbf{g}$ and scaled coupling constants $p(\lambda)$, whereas the field on the right side of this equation is defined in terms of the unscaled metric $\mathbf{g}$ and unscaled coupling constants $p$. The higher order Wick powers and their time-ordered-products have an "almost" homogeneous scaling behavior in the sense that

$$
\begin{array}{rl}
\sigma_{\lambda}\left(T\left(\prod_{i=1}^{n} \Phi_{i}\left(f_{i}\right)\right)\right)=\lambda^{-d_{T}} & T\left(\prod_{i=1}^{n} \Phi_{i}\left(f_{i}\right)\right)+ \\
\lambda^{-d_{T}} \sum_{P} T & \left(\prod_{I \in P} O_{|I|}\left(\lambda ; \times_{j \in I} f_{j} \Phi_{j}\right) \prod_{i \notin I \forall I \in P} \Phi_{i}\left(f_{i}\right)\right),
\end{array}
$$

where $d_{T}$ is the engineering dimension of the time-ordered-product and the quantities

$$
O_{n}\left(\lambda ; \times_{i=1}^{n} f_{i} \Phi_{i}\right)=\sum_{j} F_{j}(\log \lambda) \Psi_{j}
$$

have the same properties as the quantities eq. (20) in our uniqueness theorem, with the only difference that the scalar densities $F_{j}(\log \lambda)$ now have an additional polynomial dependence on $\log \lambda$.

As we will see, the fields in the interacting quantum field theory will not have this almost homogeneous scaling behavior in general.

\footnotetext{
${ }^{6}$ The fact that the non-homogeneous terms on the right side of eq. (30) take the form of local, covariant fields that depend polynomially on $\log \lambda$ was taken as an axiom in 14 , the consistency of which was proven in [15]. The specific form of these terms follows from the uniqueness theorem of [14].
} 


\section{Interacting fields in curved spacetime}

\subsection{Definition of the interacting field}

In this section, we consider the interacting field theory described by the Lagrangian density (1). Our main aim is to define the interacting field, $\varphi_{L_{1}}$, as well as its Wick powers and the time-ordered-products of its Wick powers. We use the generic notation $\Phi_{L_{1}}$ to denote any Wick power and $T_{L_{1}}\left(\prod \Phi_{i}\right)$ to denote any time-ordered-product of Wick powers of the interacting field.

The first step is to define a suitable algebra, $\mathcal{X}(M, \mathbf{g})$, of which these interacting fields will be elements. The interacting field algebra will then be defined to be a suitable subalgebra, $\mathcal{B}_{L_{1}}(M, \mathbf{g})$, of $\mathcal{X}(M, \mathbf{g})$ (see eq. (46) below). Unfortunately, even in Minkowski spacetime, if $\kappa \neq 0$ there is no known way to construct the fields for this theory other than on the level of perturbation theory. Furthermore, the perturbative formulae for the quantities that are normally calculated - such as Green's functions and S-matrix elements - are not expected to converge. In this regard, however, we note that quantities such as Green's functions and $S$-matrix elements do not depend solely on the algebraic properties of the fields themselves, but also involve properties of the vacuum state or ground state and, in many instances, also "in" and "out" states. However, even if, in some suitable sense, the algebra of fields were to vary analytically under changes of the parameter $\kappa$, there is no reason that certain states of the theory, such as the ground state, need vary analytically. This suggests the possibility that if perturbation theory were used solely for the purpose of calculating algebraic relations involving the interacting field-rather than properties involving states - then perhaps at least some of the difficulties with the convergence of perturbative expansions would not arise. In other words, rather than using perturbation

theory to calculate Green's functions, $S$-matrix elements, or other quantities that depend upon states, we suggest that it may be more fruitful to use perturbation theory to attempt to find analytic relations between the field observables that hold to all orders in perturbation theory.

However, we shall not attempt to pursue any such program here, but rather will only attempt to construct the interacting theory at the level of formal power series in the coupling constant $\kappa$. Thus, we shall take $\mathcal{X}(M, \mathbf{g})$ to be

$$
\mathcal{X}(M, \mathbf{g})=\chi_{n=0}^{\infty} \mathcal{W}(M, \mathbf{g})
$$

where an element $A \in \mathcal{X}(M, \mathbf{g})$ of the form $A=\left(A_{0}, A_{1}, A_{2}, \ldots\right)$ should be interpreted as corresponding to the formal power series

$$
A=\sum_{n=0}^{\infty} A_{n} \kappa^{n} .
$$

The multiplication law in $\mathcal{X}(M, \mathbf{g})$ is then defined to be that corresponding to the multiplication of the formal power series expressions (33), i.e., if $A=\left(A_{0}, A_{1}, A_{2}, \ldots\right)$ and 
$B=\left(B_{0}, B_{1}, B_{2}, \ldots\right)$, then $A \star B=\left(A_{0} \star B_{0}, A_{1} \star B_{0}+A_{0} \star B_{1}, \ldots\right)$. Note that the interacting field algebra $\mathcal{B}_{L_{1}}(M, \mathbf{g}) \subset \mathcal{X}(M, \mathbf{g})$ that we will define in eq. (46) below will then formally correspond to the entire one parameter family of interacting field algebras for all values of $\kappa$, rather than the interacting field algebra for a specific value of $\kappa$.

To define the interacting field, we first consider a situation in which the interaction is turned on only in some finite spacetime region, i.e., we choose a cutoff function, $\theta$, of compact support on $M$ which is equal to 1 on an open neighborhood of the closure, $\bar{V}$, of some globally hyperbolic open region $V$ with the property that $\Sigma \cap V$ is a Cauchy surface for $V$ for some Cauchy surface $\Sigma$ in $M$. This cutoff will be removed in a later step (see below). We define the relative $S$-matrix for $f \Phi$ with respect to the interaction Lagrangian density $\theta \boldsymbol{L}_{1}$ by

$$
\mathcal{S}_{\theta L_{1}}(f \Phi)=S\left(\theta \boldsymbol{L}_{1}\right)^{-1} \star S\left(\theta \boldsymbol{L}_{1}+f \Phi\right)
$$

where the local $S$-matrix, $S(f \Phi)$, was defined in eq. (15) above. Then the Wick power, $\Phi_{\theta L_{1}}$, for the interacting theory with Lagrangian density $\theta \boldsymbol{L}_{1}$ corresponding to the Wick power $\Phi$ of the free theory is defined by [2]

$$
\left.\Phi_{\theta L_{1}}(f) \equiv \frac{\partial}{i \partial \alpha} \mathcal{S}_{\theta L_{1}}(\alpha f \Phi)\right|_{\alpha=0} .
$$

Here the right side of eq. (35) should be viewed as (rigorously) defining an element of $\mathcal{X}(M, \mathbf{g})$, which is obtained by formally expanding $S\left(\theta \boldsymbol{L}_{1}\right)^{-1}$ and $S\left(\theta \boldsymbol{L}_{1}+f \Phi\right)$ in powers of the coupling constant $\kappa$ and then collecting all of the (finite number of) terms that multiply $\kappa^{n}$ for each $n$ (see eq. (33) above and eq. (37) below). Similarly, the time-orderedproduct of Wick powers of the interacting field with Lagrangian density $\theta \boldsymbol{L}_{1}$ is defined by

$$
\left.T_{\theta L_{1}}\left(\prod_{i=1}^{n} \Phi_{i}\left(f_{i}\right)\right) \equiv \frac{\partial^{n}}{i^{n} \partial \alpha_{1} \ldots \partial \alpha_{n}} \mathcal{S}_{\theta L_{1}}\left(\sum_{i} \alpha_{i} f_{i} \Phi_{i}\right)\right|_{\alpha_{1}=\cdots=\alpha_{n}=0} .
$$

Note that the definition of $\Phi_{\theta L_{1}}$ (as well as that of $T_{\theta L_{1}}\left(\prod \Phi_{i}\right)$ ) has been adjusted so that $\Phi_{\theta L_{1}}$ coincides with the corresponding free field $\Phi$ before the interaction is "switched on". This can be seen explicitly by expressing $\Phi_{\theta L_{1}}(f)$ in terms of the "totally retarded products"

$$
\Phi_{\theta L_{1}}(f)=\Phi(f)+\sum_{n \geq 1} \frac{i^{n}}{n !} R(f \Phi ; \underbrace{\theta \boldsymbol{L}_{1}, \ldots, \theta \boldsymbol{L}_{1}}_{n \text { factors }}),
$$

Since the $R$-products have support

$$
\operatorname{supp} R \subset\left\{\left(y, x_{1}, \ldots, x_{n}\right) \mid x_{i} \in J^{-}(y) \quad \forall i\right\},
$$

${ }^{7}$ This formula is known as "Haag's series," since an expansion of this kind was first derived in 12 for Minkowski spacetime; see also [11]. 
it follows that all terms in the above sum will vanish if the support of $f$ does not intersect the causal future of the support of $\theta$.

Below, we will need to know how the fields (36) change under a change of the cutoff function $\theta$. Now if $\theta$ and $\theta^{\prime}$ are two cutoff functions, each of which are 1 in an open neighborhood of $\bar{V}$ as above, then there exists a smooth function $h_{-}$of compact support on $M$ which is equal to $\theta-\theta^{\prime}$ on the causal past of the region $V$, and whose support does not intersect the causal future of $V$. The unitary $U\left(\theta, \theta^{\prime}\right)$ defined by

$$
U\left(\theta, \theta^{\prime}\right)=\mathcal{S}_{\theta L_{1}}\left(h_{-} \boldsymbol{L}_{1}\right)
$$

is then independent of the particular choice for $h_{-}$, and one has [四, thm. 8.6]

$$
U\left(\theta, \theta^{\prime}\right) \star T_{\theta L_{1}}\left(\prod \Phi_{i}\left(f_{i}\right)\right) \star U\left(\theta, \theta^{\prime}\right)^{-1}=T_{\theta^{\prime} L_{1}}\left(\prod \Phi_{i}\left(f_{i}\right)\right)
$$

for all fields $\Phi_{i}$ and all smooth scalar densities $f_{i}$ of compact support in $V$.

We now remove the cutoff $\theta$. Formulas (35) and (36) will not, in general, make sense if we straightforwardly attempt to take the limit $\theta \rightarrow 1$. Indeed if $\theta$ could be set equal to 1 throughout the spacetime in eq. (35), then the resulting formula for $\Phi_{L_{1}}$ would define an interacting field in the sense of Bogoliubov [2], with the property that the interacting field approaches the free field in the asymptotic past. However, even in Minkowski spacetime, it is far from clear that such an asymptotic limit of the interacting field will exist (particularly for massless fields), and it is much less likely that any such limit would exist in generic globally hyperbolic curved spacetimes that are not flat in the asymptotic past.

In order to remove the cutoff in a manner in which the limit will exist, we will not try to take a limit where the field remains fixed in the asymptotic past but rather-following the ideas of [⿴囗口 — - we will take a limit where the field remains fixed in regions of increasing size in the interior of the spacetime. To make this construction precise, it is useful to have the following lemma:

Lemma 3.1. Let $(M, \mathbf{g})$ be a globally hyperbolic spacetime. Then there exists a sequence of compact sets, $\left\{K_{n}\right\}$, with the properties that (i) for each $n, K_{n} \subset V_{n+1}$, where $V_{n+1} \equiv$ $\operatorname{int}\left(K_{n+1}\right)$ (ii) $\cup_{n} K_{n}=M$, and (iii) for each $n, V_{n}$ is globally hyperbolic and $\Sigma \cap V_{n}$ is a Cauchy surface for $V_{n}$, where $\Sigma$ is a Cauchy surface for $M$.

Proof. Let $t$ be a time function on $(M, \mathbf{g})$ with range $-\infty<t<\infty$ whose level surfaces are Cauchy surfaces, $\Sigma_{t}$, that foliate $M$ [10], [7]. Let $\Sigma=\Sigma_{0}$. Choose any complete Riemannian metric, $q_{a b}$, on $\Sigma$, choose $x_{0} \in \Sigma$, and let $B_{n}$ be the closed ball (on $\Sigma$ ) of radius $n$ about $x_{0}$ with respect to $q_{a b}$. Define

$$
K_{n}=D\left(B_{n}\right) \cap J^{-}\left(\Sigma_{n}\right) \cap J^{+}\left(\Sigma_{-n}\right)
$$

where $D$ denotes the domain of dependence and $J^{-}$and $J^{+}$denote the causal past and future, respectively. Then $K_{n}$ is closed. Furthermore, since $B_{n}$ is compact it follows that 
$J^{+}\left(B_{n}\right) \cap J^{-}\left(\Sigma_{n}\right)$ and $J^{-}\left(B_{n}\right) \cap J^{+}\left(\Sigma_{-n}\right)$ are compact. Since $K_{n}$ is a subset of the union of these two sets, it follows that $K_{n}$ is compact. Clearly, we have $V_{n} \subset V_{n+1}$. However, if $x$ lies on the boundary of $K_{n}$, then it must lie on the boundary of $D\left(B_{n}\right)$ and/or lie on $\Sigma_{n}$ or $\Sigma_{-n}$; in all cases, it follows immediately that $x \in V_{n+1}$. Thus, $K_{n} \subset V_{n+1}$. To prove property (ii), let $y \in M$ with, say, $y \in J^{+}(\Sigma)$. Since $J^{-}(y) \cap \Sigma$ is compact, it must be contained in some ball of radius $r$ about $x_{0}$ (with respect to the metric $q_{a b}$ on $\Sigma$ ). Then $y \in D\left(B_{r}\right)$, so $y \in K_{n}$ for any $n$ such that $n>r$ and $n>t(y)$, as we desired to show. Finally, the fact that $V_{n}$ is globally hyperbolic with Cauchy surface $V_{n} \cap \Sigma$ follows immediately from the fact that $V_{n}$ is the interior of the domain of dependence of $B_{n}$ for the spacetime $I^{-}\left(\Sigma_{n}\right) \cap I^{+}\left(\Sigma_{-n}\right)$.

Let $\left\{K_{n}\right\}, n=1,2, \ldots$, be a sequence of compact sets with the properties stated in lemma 3.1. For each $n$, let $\theta_{n}$ be a smooth function with support contained in $K_{n+1}$ such that $\theta_{n}=1$ on an open neighborhood of $K_{n}$. Let $U_{1}=\mathbb{1}$ and let $U_{n}=U\left(\theta_{n}, \theta_{n-1}\right)$ for all $n>1$, where $U\left(\theta_{n}, \theta_{n-1}\right)$ was defined in eq. (39) above. Write $u_{n}=U_{1} \star U_{2} \star \cdots \star U_{n}$. Our definition of the interacting field, its Wick powers, and their time-ordered-products is:

$$
T_{L_{1}}\left(\prod \Phi_{i}\left(f_{i}\right)\right) \equiv \lim _{n \rightarrow \infty} \operatorname{Ad}\left(u_{n}\right) T_{\theta_{n} L_{1}}\left(\prod \Phi_{i}\left(f_{i}\right)\right),
$$

where we use the notation $\operatorname{Ad}\left(u_{n}\right) A=u_{n} \star A \star u_{n}^{-1}$ for any $A \in \mathcal{X}(M, \mathbf{g})$. The existence of the limit is a direct consequence of the following proposition:

Proposition 3.1. Suppose that $N$ is such that the support of each $f_{i}$ is contained in $K_{N}$. Then for all $n, m \geq N$ we have

$$
\operatorname{Ad}\left(u_{n}\right) T_{\theta_{n} L_{1}}\left(\prod \Phi_{i}\left(f_{i}\right)\right)=\operatorname{Ad}\left(u_{m}\right) T_{\theta_{m} L_{1}}\left(\prod \Phi_{i}\left(f_{i}\right)\right)
$$

Proof. It suffices to show that for any $n \geq N$ we have

$$
u_{n+1} \star T_{\theta_{n+1} L_{1}}\left(\prod \Phi_{i}\left(f_{i}\right)\right) \star u_{n+1}^{-1}=u_{n} \star T_{\theta_{n} L_{1}}\left(\prod \Phi_{i}\left(f_{i}\right)\right) \star u_{n}^{-1}
$$

But by eq. (40) we have

$$
U_{n+1} \star T_{\theta_{n+1} L_{1}}\left(\prod \Phi_{i}\left(f_{i}\right)\right) \star U_{n+1}^{-1}=T_{\theta_{n} L_{1}}\left(\prod \Phi_{i}\left(f_{i}\right)\right)
$$

from which the desired result follows immediately by applying $\operatorname{Ad}\left(u_{n}\right)$ to both sides.

Now, given any compact set $K \subset M$ and any family of compact sets $K_{n}$ satisfying properties (i) and (ii) of the above lemma, then there always exists an $N$ such that

\footnotetext{
${ }^{8}$ Proof: Otherwise, one could find a sequence $\left\{x_{n}\right\} \in K$ such that $x_{n} \notin K_{n}$ for all $n$. However, this sequence would have an accumulation point, $x$, which must lie in the interior of some $K_{N}$, resulting in a contradiction.
} 
$K \subset K_{N}$. Given any smeared time-ordered-product of Wick powers, we choose $K$ to be the union of the supports of all of the (finite number of) test functions appearing in the time-ordered product. By the above proposition, there exists an $N$ such that the sequence appearing on the right side of eq. (42) is constant for all $n>N$. Therefore, the limit exists.

The meaning of the sequence $\operatorname{Ad}\left(u_{n}\right) T_{\theta_{n} L_{1}}\left(\prod \Phi_{i}\left(f_{i}\right)\right), n=1,2, \ldots$, is easily understood as follows. Since $u_{1}=\mathbb{1}$, the first element of this sequence is just the Bogoluibov formula for this interacting field quantity with cutoff function $\theta_{1}$. The second element of this sequence modifies the Bogoliubov formula with cutoff function $\theta_{2}$ in such a way that, according to eq. (40) above, the modified Bogoliubov formula with cutoff function $\theta_{2}$ agrees with the unmodified Bogoliubov formula with cutoff function $\theta_{1}$ when the supports of all of the $f_{i}$ are contained within $K_{1}$. For the third element of the sequence, the unitary map $U_{3}$ first modifies the Bogoliubov formula with cutoff function $\theta_{3}$ so that it agrees in region $K_{2}$ with the Bogoliubov formula with cutoff function $\theta_{2}$. The action of the unitary $U_{2}$ then further modifies this expression so that it agrees in region $K_{2}$ with the modified Bogoliubov formula of the previous step. In this way, we have implemented the idea of "keeping the interacting field fixed in the interior of the spacetime" as the cutoff is removed.

We define the interacting field algebra $\mathcal{B}_{L_{1}}(M, \mathbf{g})$ to be the subalgebra of $\mathcal{X}(M, \mathbf{g})$ generated by the interacting field, its Wick powers, and their time-ordered-products, i.e.,

$$
\mathcal{B}_{L_{1}}(M, \mathbf{g}) \equiv\left\{\text { algebra generated by } T_{L_{1}}\left(\prod \Phi_{i}\left(f_{i}\right)\right) \mid f_{i} \in \mathcal{D}_{1}(M), \Phi_{i} \in \mathcal{V}\right\} .
$$

This definition of $\mathcal{B}_{L_{1}}(M, \mathbf{g})$ as a subalgebra of $\mathcal{X}(M, \mathbf{g})$ depends on a choice of a family of compact sets $K_{n}$ satisfying the properties of lemma 3.1 as well as a choice of cutoff functions $\theta_{n}$. If we were to choose a different family, $\tilde{K}_{n}$, of compact sets and a corresponding different family, $\tilde{\theta}_{n}$, of cutoff functions, we will obtain a different subalgebra $\tilde{\mathcal{B}}_{L_{1}}(M, \mathbf{g}) \subset \mathcal{X}(M, \mathbf{g})$ of interacting fields. However, the algebra $\tilde{\mathcal{B}}_{L_{1}}(M, \mathbf{g})$ is isomorphic to $\mathcal{B}_{L_{1}}(M, \mathbf{g})$. To see this, focus attention on the subalgebras $\tilde{\mathcal{B}}_{L_{1}}(K, \mathbf{g})$ and $\mathcal{B}_{L_{1}}(K, \mathbf{g})$ generated by fields that are smeared with test functions with support in a fixed compact set $K$. Let $n$ be such that $K \subset K_{n}$ and $K \subset \tilde{K}_{n}$. Let

$$
X_{n}=u_{n} \star U\left(\tilde{\theta}_{n}, \theta_{n}\right) \star \tilde{u}_{n}^{-1} .
$$

Then $X_{n}$ is a unitary element of $\mathcal{X}(M, \mathbf{g})$. However, for any $\tilde{F} \in \tilde{\mathcal{B}}_{L_{1}}(K, \mathbf{g})$, it follows from eqs. (40) and (42) together with proposition 3.1 that $\operatorname{Ad}\left(X_{n}\right) \tilde{F}$ is the corresponding interacting field quantity $F \in \mathcal{B}_{L_{1}}(K, \mathbf{g})$. This shows that the map $\gamma_{K}: \tilde{\mathcal{B}}_{L_{1}}(K, \mathbf{g}) \rightarrow$ $\mathcal{B}_{L_{1}}(K, \mathbf{g})$ which associates to any element of $\tilde{\mathcal{B}}_{L_{1}}(K, \mathbf{g})$ the corresponding interacting field quantity in $\mathcal{B}_{L_{1}}(K, \mathbf{g})$ is well defined and is a ${ }^{*}$-isomorphism. However, since $K$ is arbitrary, this argument actually shows that the map $\gamma: \tilde{\mathcal{B}}_{L_{1}}(M, \mathbf{g}) \rightarrow \mathcal{B}_{L_{1}}(M, \mathbf{g})$ which associates to any element of $\tilde{\mathcal{B}}_{L_{1}}(M, \mathbf{g})$ the corresponding element of $\mathcal{B}_{L_{1}}(M, \mathbf{g})$ also is 
well defined and is a ${ }^{*}$-isomorphism of these algebras \%. Thus, as an abstract algebra, $\mathcal{B}_{L_{1}}(M, \mathbf{g})$ is independent of the choices of $K_{n}$ and $\theta_{n}$ that entered in its construction. In the following we assume that we have made an arbitrary, but fixed, choice for $K_{n}$ and $\theta_{n}$ in every spacetime.

In the free theory, the notion of a local and covariant field was defined relative to a natural injective ${ }^{*}$-homomorphism $\alpha_{\chi}: \mathcal{W}(\tilde{M}, \tilde{\mathbf{g}}) \rightarrow \mathcal{W}(M, \mathbf{g})$ associated with causality preserving isometric embeddings $\chi$ of a spacetime $(\tilde{M}, \tilde{\mathbf{g}})$ into another spacetime $(M, \mathbf{g})$. The Wick products of the free field and their time-ordered-products were then seen to be local, covariant fields in the sense that eq. (10) holds. In order to get a corresponding natural injective ${ }^{*}$-homomorphism, $\boldsymbol{\alpha}_{\chi}: \mathcal{B}_{L_{1}}(\tilde{M}, \tilde{\mathbf{g}}) \rightarrow \mathcal{B}_{L_{1}}(M, \mathbf{g})$, for the interacting field algebra, we must compose the natural action of $\alpha_{\chi}$ on $\mathcal{B}_{L_{1}}(\tilde{M}, \tilde{\mathbf{g}})$ with the map $\gamma$ constructed above in order to compensate for the fact that the choices for $K_{n}$ and $\theta_{n}$ on $(M, \mathbf{g})$ may not correspond to the choices of $\tilde{K}_{n}$ and $\tilde{\theta}_{n}$ on $(\tilde{M}, \tilde{\mathbf{g}})$. It then follows that the interacting field, its Wick powers and their time-ordered-products as defined above are local and covariant fields in the sense that for any causality preserving isometric embedding, $\chi$, we have

$$
\boldsymbol{\alpha}_{\chi}\left(\Phi_{L_{1}}[\tilde{M}, \tilde{\mathbf{g}}](x)\right)=\Phi_{L_{1}}[M, \mathbf{g}](\chi(x))
$$

with an analogous equation holding for the interacting time-ordered-products.

Finally, we comment upon how the theory we have just defined is to be interpreted, i.e., how the mathematical formulas derived above for the interacting field relate to predictions of physically observable phenomena. In many discussions of quantum field theory in Minkowski spacetime, the interpretation of the theory is made entirely via the (global) $S$-matrix. Here it is assumed that in the asymptotic past and future, states of the field can be identified with states of a free field theory, which have a natural particle interpretation. It is also assumed that one can prepare states corresponding to desired incoming particle states and that one can measure the properties of the state of outgoing particles, so that the $S$-matrix can be determined. A wide class of predictions of the theory-including essentially all of the ones that can be measured in practice - can thereby be formulated in terms of measurements of the $S$-matrix for particle scattering, without the need to even mention local fields. Indeed, when this viewpoint on quantum field theory is taken to the extreme, the local quantum fields, in effect, play the role of merely being tools used for calculating the $S$-matrix.

An alternative, but closely related, viewpoint on interpreting the theory in Minkowski spacetime makes crucial use of the existence of a preferred vacuum state. Here, one focuses attention on the correlation functions of the field in this state, which are assumed to be measureable - at least in the asymptotic past and future and for sufficiently large spatial separation of the points. The interpretation of the theory can be formulated in terms of

\footnotetext{
${ }^{9}$ Note, however, that there need not exist a unitary element $X \in \mathcal{X}(M, \mathbf{g})$ whose action on $\tilde{\mathcal{B}}_{L_{1}}(M, \mathbf{g})$ coincides with $\gamma$.
} 
its predictions for these correlation functions. This viewpoint on the interpretation of the theory is closely related to the first one, since the particle measurements in the $S$-matrix interpretation can be viewed as really corresponding to measuring certain properties of these correlation functions.

However, for quantum fields in a general, globally hyperbolic curved spacetime, we do not expect to have asymptotic, free particle states or any globally preferred states. It therefore would not appear fruitful to attempt to interpret the theory in a manner analogous to the above ways in which the theory is normally interpreted in Minkowski spacetime. Rather, it would seem much more fruitful to view the interacting field itselftogether with its Wick powers and other local covariant fields in $\mathcal{B}_{L_{1}}(M, \mathbf{g})$ - as the fundamental observables in the theory. To make "measurements", we assume that we have access to some external systems that couple to the field observables of interest via known interaction Lagrangians, and that we can then measure the state of the external systems at different times. It is clear that by making sufficiently many measurements of this sort, we can test any aspect of the theory and - if the theory is valid - we also can determine any unknown coupling parameters in the theory. However, it is not straightforward to give a simple, universal algorithm for doing so, since the properties of the states will depend upon the spacetime under consideration, and a type of experiment that would most usefully probe the theory for a particular spacetime may not be as useful for another spacetime.

To make the remarks of the previous paragraph more explicit, consider a typical experiment in Minkowski spacetime wherein one prepares a system of particles in a given incoming state and measures the particle content of the outgoing particles. Both the "state preparation" and the "measurement" of the "particles" in their final state really consist of introducing certain external systems that have desired couplings to the quantum field, preparing the initial state of these external systems suitably, and measuring their final state. In a curved spacetime, one could presumably introduce external systems with couplings to the field that are similar to those of systems used in Minkowski spacetime, although it should be noted that there is not any obvious, general notion of what it means to have "the same" system in a curved spacetime as one had in Minkowski spacetime, unless one goes to a limit where the size of the system is much smaller than any curvature scales. However, even if one considers an external system in curved spacetime that corresponds to a system of "particle detectors" in Minkowski spacetime, it may not be possible to give any consistent interpretation of the outcome of the curved spacetime measurements in terms of "particles". Nevertheless, such measurements still provide information about the states of the quantum field, and it is clear that all aspects of the quantum field theory can be probed by coupling the field to suitable external systems and measuring the state of these external systems.

In should be noted that the above situation is not significantly different from the case of classical field theory. Suppose that a classical field $\varphi$ with Lagrangian (1) can be measured via its effect on the motion of scalar test charges, which feel a force proportional 
to $\nabla_{a} \varphi$. In Minkowski spacetime, one could set up an experiment where a global family of inertial observers release test particles at some time in the distant past. By studying the test particle motion for a brief interval of time, they could reconstruct $\varphi$ (up to a constant) in that region of spacetime and associate a noninteracting solution with the state of the field in the distant past. By repeating this procedure in the distant future they could obtain a corresponding non-interacting solution there, and they could thereby determine the classical $S$-matrix. A great deal of information about the interacting theory is encoded in the classical $S$-matrix. However, it does not seem straightforward to give a simple algorithm for making measurements with a similar interpretative content in a general curved spacetime, where there are no asymptotic regions and no globally preferred families of observers. Nevertheless, it is clear that the classical field theory in curved spacetime is as meaningful and interpretable as in Minkowski spacetime, and that all of the predictions of the curved spacetime theory can be probed by doing experiments that study the motion of a sufficiently wide class of test particles.

\subsection{Renormalization ambiguities for the interacting field}

In the previous subsection we explained the construction of the interacting Wick products and their time-ordered-products in the interacting field theory classically described by the Lagrangian $\boldsymbol{L}$ given by (1). These constructions were based on a prescription for defining the Wick products and their time-ordered-products in the corresponding free field theory. As we discussed in section 2, the definition of these quantities is subject to some wellspecified ambiguities. Therefore, the quantities in the interacting field theory also will be subject to ambiguities.

The purpose of this section is to give a precise specification of these ambiguities. We shall show is that a change in the prescription for the Wick products and their timeordered-products (within the class of "allowed prescriptions" specified by our uniqueness theorem) corresponds to a shift of coupling parameters of the theory appearing in the Lagrangian (1). More precisely, the interacting field algebra obtained with the new prescription will be isomorphic to the interacting field algebra obtained with the original prescription, but with the interaction Lagrangian modified by the addition of "counterterms", which - for a renormalizable theory, as considered here - are of the same form as those appearing in the original Lagrangian. This isomorphism of the interacting field algebras for the two different prescriptions will map the interacting field to a multiple of the interacting field. However, the relationship between the higher Wick powers of the interacting field and their time-ordered-products as defined by the two prescriptions is more complicated: the isomorphism between the algebras will map a higher Wick power (or a time-ordered-product of Wick powers) into a field of the form specified in eq. (52) below.

To make the above statements more explicit, suppose that we are given two prescriptions for defining the Wick products and their time ordered products in the free field 
theory, both satisfying the assumptions of our uniqueness theorem. These prescriptions will give rise to two different constructions of interacting fields, which we shall denote as $T_{L_{1}}\left(\prod \Phi_{i}\right)$ respectively $\tilde{T}_{L_{1}}\left(\prod \tilde{\Phi}_{i}\right)$, and we write $\mathcal{B}_{L_{1}}(M, \mathbf{g})$ respectively $\tilde{\mathcal{B}}_{L_{1}}(M, \mathbf{g})$ for the algebras generated by these fields. Then the relation between the tilde interacting fields and the untilde interacting fields can be stated as follows: There exists a ${ }^{*}$-isomorphism

$$
r: \tilde{\mathcal{B}}_{L_{1}}(M, \mathbf{g}) \rightarrow \mathcal{B}_{L_{1}+\delta L_{1}}(M, \mathbf{g})
$$

such that

$$
r\left(\tilde{\varphi}_{L_{1}}(f)\right)=Z \varphi_{L_{1}+\delta L_{1}}(f),
$$

for all $f \in \mathcal{D}_{1}(M)$. The field $\tilde{\varphi}_{L_{1}}$ on the left side of eq. (50) is the interacting field defined using the "tilde prescription" with respect to the interaction Lagrangian density $\boldsymbol{L}_{1}$, whereas the field $\varphi_{L_{1}+\delta L_{1}}$ on the right side of this equation is defined using the "untilde prescription" with respect to the interaction Lagrangian density $\boldsymbol{L}_{1}+\delta \boldsymbol{L}_{1}$, where $\delta \boldsymbol{L}_{1}$ is given by

$$
\delta \boldsymbol{L}_{1}=\frac{1}{2}\left[\delta z(\nabla \varphi)^{2}+\delta \xi R \varphi^{2}+\delta m^{2} \varphi^{2}+\delta \kappa \varphi^{4}\right] \boldsymbol{\epsilon} .
$$

The parameters in this expression (including $\delta \kappa$ ), as well the parameter $Z$ in eq. (50) are formal power series in $\kappa$ with real coefficients. The generalization of formula (50) for the action of $r$ on an arbitrary interacting time-ordered-product in the tilde prescription is given by

$$
\begin{aligned}
r\left(\tilde{T}_{L_{1}}\left(\prod_{i=1}^{n} \tilde{\Phi}_{i}\left(f_{i}\right)\right)\right)=T_{L_{1}+\delta L_{1}}\left(\prod_{i=1}^{n} Z_{i} \Phi_{i}\left(f_{i}\right)\right)+ \\
\sum_{P} T_{L_{1}+\delta L_{1}}\left(\prod_{I \in P} \boldsymbol{O}_{|I|}\left(\times_{i \in I} f_{i} \Phi_{i}\right) \prod_{j \notin I \forall I \in P} Z_{j} \Phi_{j}\left(f_{j}\right)\right) .
\end{aligned}
$$

Here, the $Z_{i}$ are formal power series in $\kappa$ whose coefficients are real provided the corresponding field $\Phi_{i}$ is (formally) hermitian. The $\boldsymbol{O}_{n}$ are multilinear maps from $\times{ }^{n} \mathcal{D}_{1}(M, \mathcal{V}) \rightarrow$ $\mathcal{D}_{1}(M, \mathcal{V})$ that depend on the interaction Lagrangian $\boldsymbol{L}_{1}$ and have similar properties to the maps $O_{n}$ in our uniqueness theorem for the time-ordered products of Wick products in the free theory: First, the $\boldsymbol{O}_{n}$ can be given an analogous representation to the quantities $O_{n}$ in the free theory given in eq. (19),

$$
\boldsymbol{O}_{n}\left(\times_{i=1}^{n} f_{i} \Phi_{i}\right)=\sum_{j} c_{j} G_{j} \Psi_{j}
$$

The densities $G_{j}$ have the same form as the the corresponding expressions $F_{j}$ in the free theory (see eq. (20)), and the $c_{j}$ are formal power series in $\kappa$. If the terms appearing on the right side of eq. (53) are written out in terms of geometrical tensors (and the coupling 
constants in the free theory), then the engineering dimensions of each term will satisfy a "power counting relation" identical to that in the free theory, eq. (23).

In terms of the generating functional

$$
\mathcal{S}_{L_{1}}\left(\sum f_{i} \Phi_{i}\right)=\mathbb{1}+\sum_{n \geq 1} \frac{i^{n}}{n !} T_{L_{1}}\left(\prod^{n} \sum \Phi_{i}\left(f_{i}\right)\right)
$$

for the interacting Wick products and time-ordered-products, and the generating functional

$$
\boldsymbol{\delta}_{L_{1}}\left(\sum f_{i} \Phi_{i}\right) \equiv \sum_{n \geq 1} \frac{i^{n-1}}{n !} \boldsymbol{O}_{n}\left(\chi^{n} \sum f_{i} \Phi_{i}\right)
$$

relations (52) can be rewritten more compactly as

$$
r\left(\widetilde{\mathcal{S}}_{L_{1}}\left(\sum f_{i} \Phi_{i}\right)\right)=\mathcal{S}_{L_{1}+\delta L_{1}}\left(\sum Z_{i} f_{i} \Phi_{i}+\boldsymbol{\delta}_{L_{1}}\left(\sum f_{i} \Phi_{i}\right)\right)
$$

In the preceding discussion, we have highlighted the analogies between the structure of the renormalization ambiguities in the free and interacting theories. However, there are also some key differences. Firstly, in our identity (25) specifying the renormalization ambiguities of the time-ordered-products in the free theory, the tilde and untilde time-ordered-products are defined both "with respect to the same Lagrangian". By contrast, in the corresponding formula (52) in the interacting theory, the tilde and untilde time-ordered-products are defined with respect to different Lagrangians. A second key difference between formulas (25) and (52) the free and interacting theories is the appearance of the "field strength renormalization factors," $Z_{i}$, in the interacting theory, which are absent in the free theory. Third, while the maps $O_{n}$ and $\boldsymbol{O}_{n}$ in the free and interacting theories satisfy a number of similar properities, the map $\boldsymbol{O}_{n}$ does not satisfy the commutator property, eq. (21), satisfied by $O_{n}$ in the free theory. Fourth, we note the appearance of the automorphism $r$ in our formula (52) for the renormalization ambiguity of the interacting time-ordered-products, which is absent in the corresponding formula (25) in the free theory.

Proof of equation (52): Let $\theta$ be a cutoff function of compact support as above which is 1 in an open neighborhood of the closure, $\bar{V}$ of a globally hyperbolic subset $V$ of $M$ such that $V \cap \Sigma$ is a Cauchy surface of $V$ for some Cauchy surface $\Sigma$ of $M$. Eq. (16) implies that

$$
\widetilde{\mathcal{S}}_{\theta L_{1}}(f \Phi)=S\left(\theta \boldsymbol{L}_{1}+\delta\left(\theta \boldsymbol{L}_{1}\right)\right)^{-1} \star S\left(f \Phi+\theta \boldsymbol{L}_{1}+\delta\left(f \Phi+\theta \boldsymbol{L}_{1}\right)\right) .
$$

In order to bring this equation into a more convenient form, let us define the following elements in $\mathcal{X}(M, \mathbf{g})$ :

$$
\left.\delta_{n}\left(\theta \boldsymbol{L}_{1} ; f_{1} \Phi_{1}, \ldots, f_{n} \Phi_{n}\right) \equiv \frac{\partial^{n}}{i^{n-1} \partial \alpha_{1} \ldots \partial \alpha_{n}} \delta\left(\theta \boldsymbol{L}_{1}+\sum_{i=1}^{n} \alpha_{i} f_{i} \Phi_{i}\right)\right|_{\alpha_{1}=\cdots=\alpha_{n}=0}
$$


It follows from our uniqueness theorem that we can write $\delta_{0}\left(\theta \boldsymbol{L}_{1}\right)$ as a sum (over $n$ and $j$ ) of terms of the general form

$$
F_{n, j}(x) \Psi_{j}=\boldsymbol{\epsilon}(x) \sum_{(a)=\left(a_{1}\right) \ldots\left(a_{n}\right)} C_{n, j}^{(a)}(x) \prod_{i=1}^{n} \nabla_{\left(a_{i}\right)} \theta(x) \Psi_{j}
$$

where $C^{(a)}{ }_{n, j}$ are monomials in the Riemann tensor, its derivatives, and $m^{2}$. Since $\theta \boldsymbol{L}_{1}$ has engineering dimension 4, it follows from eq. (23) that each term in (59) must have engineering dimension 4. Since $\theta \boldsymbol{L}_{1}$ is hermitian, it follows from eq. (24) that the $C_{n, j}^{(a)}$ must be real and that the fields $\Psi_{j}$ must be hermitian. We now divide the terms (59) appearing in $\delta_{0}\left(\theta \boldsymbol{L}_{1}\right)$ into a group consisting of all terms not containing any derivatives of $\theta$ and a second group of terms each containing at least one derivative of $\theta$. This gives a decomposition of $\delta_{0}\left(\theta \boldsymbol{L}_{1}\right)$ into the following two groups of terms:

$$
\delta_{0}\left(\theta \boldsymbol{L}_{1}\right)=\boldsymbol{\epsilon} \sum_{n \geq 1} \kappa^{n} \theta^{n} \sum_{j} c_{n, j} \Psi_{j}+\sum_{n \geq 1} \kappa^{n} \sum_{j} f_{n, j} \boldsymbol{\Lambda}_{j}
$$

Here, $c_{n, j}$ are real constants, $\Psi_{j}$ runs through all hermitian fields of engineering dimension 4 (including fields with dimensionful couplings such as $m^{2} \varphi^{2}$ or $R^{2} \mathbb{1}$ ), the $f_{n, j}$ are compactly supported smooth densities on $M$ whose support does not intersect on open neighborhood of $\bar{V}$, and $\boldsymbol{\Lambda}_{j}$ are hermitian fields of engineering dimension less than 4 . In the decomposition (60), we may replace the smooth functions $\theta^{n}$ in the first sum by the function $\theta$ at the expense of adding new terms of the kind appearing in the second sum, except that these new terms will have engineering dimension equal to 4 . If this is done, we obtain the decomposition

$$
\delta_{0}\left(\theta \boldsymbol{L}_{0}\right)=\theta \delta \boldsymbol{L}_{1}+\sum_{j} h_{j} \boldsymbol{\Lambda}_{j}
$$

Here $\delta \boldsymbol{L}_{1}$ is the real linear combination $\boldsymbol{\epsilon} \sum a_{j} \Psi_{j}$ where $\Psi_{j}$ is running over all hermitian fields of engineering dimension 4 (including again fields with dimensionful coupling) and where $a_{j}=\sum_{n \geq 1} c_{n, j} \kappa^{n}$. The second sum in the above decomposition (61) of $\delta_{0}\left(\theta \boldsymbol{L}_{1}\right)$ contains only real test densities $h_{j}$ of compact support that vanish on an open neighborhood of $\bar{V}$. The quantities $\boldsymbol{\Lambda}_{j}$ are now hermitian fields of engineering dimension $\leq 4$.

The field (density) $\delta \boldsymbol{L}_{1}$ in eq. (61) is therefore of the form claimed in eq. (51), except that it may contain (i) terms of the form $C_{j} \mathbb{1}$, where $C_{j}$ is a monomial in the Riemann tensor, its covariant derivatives and $m^{2}$, and (ii) a term proportional to $\varphi \nabla^{a} \nabla_{a} \varphi$. In principle these terms should be included in eq. (51). However, the terms (i) proportional to the identity do not contribute to the relative $S$-matrix given by eq. (57) and can therefore be dropped. Furthermore, it can be seen that the term (ii) can always be eliminated in favor of the term $m^{2} \varphi^{2}+\xi R \varphi^{2}$ together with a sum of products of curvature tensors and 
$m^{2}$ of engineering dimension 4 times the identity $\mathbb{1}$, if the following additional condition is imposed on the time-ordered-products:

$$
T\left(\varphi\left(\nabla^{a} \nabla_{a}-m^{2}-\xi R\right) \varphi\left(f_{0}\right) \prod_{i=1}^{n} \Phi_{i}\left(f_{i}\right)\right)=T\left(\sum_{j} K_{j} \mathbb{1}\left(f_{0}\right) \prod_{i=1}^{n} \Phi_{i}\left(f_{i}\right)\right)
$$

for all $\Phi_{i}$ and all $f_{i} \in \mathcal{D}_{1}(M)$, where $K_{j}$ are monomials in the Riemann tensor, its derivatives and $m^{2}$ of engineering dimension 4 . For the case of the Wick power $\varphi\left(\nabla^{a} \nabla_{a}-\right.$ $\left.m^{2}-\xi R\right) \varphi$ itself, this condition was shown to hold by Moretti [17, eq. (47)] for the "local normal ordering prescription" given in [14] and eq. (105) below. Using the methods of [15], it can be shown that this additional normalization condition can also be satisfied for general time-ordered-products of the form (62). Therefore, we will assume that a condition of the form eq. (62) has been imposed 0 . It then follows that $\delta \boldsymbol{L}_{1}$ has the form claimed in eq. (51).

Again, using the properties of the maps $O_{n}$ in our uniqueness theorem, we can write

$$
\delta_{1}\left(\theta \boldsymbol{L}_{1} ; f \Phi\right)=f \delta Z \Phi+\boldsymbol{O}_{1}(f \Phi)
$$

where $\delta Z$ is a formal power series in the coupling constant $\kappa$. If $\Phi$ is hermitian, then it follows again from eq. (24) that these power series have real coefficients. The element $\boldsymbol{O}_{1}(f \Phi)$ is of the form $\sum Z_{j} G_{j} \Psi_{j}$, where the $G_{j}$ can be written as

$$
G_{j}(x)=\boldsymbol{\epsilon}(x) \sum_{(a)} C_{j}^{(a)}(x) \nabla_{(a)} f(x)
$$

where we have identified the density $f$ with a smooth function on $M$ via the metric volume element $\boldsymbol{\epsilon}$ and where the $C_{j}{ }^{(a)}$ are monomials in the Riemann tensor, its derivatives and $m^{2}$ of the correct dimension. The $Z_{j}$ are formal power series in $\kappa$ and the $\Psi_{j}$ are local covariant fields with fewer powers in the free field than $\Phi$. Moreover, for $n \geq 2$, we define

$$
\boldsymbol{O}_{n}\left(\times_{i=1}^{n} f_{i} \Phi_{i}\right) \equiv \delta_{n}\left(\theta \boldsymbol{L}_{1} ; f_{1} \Phi_{1}, \ldots, f_{n} \Phi_{n}\right) .
$$

Using the properties of $O_{n}$ given in our uniqueness theorem for the time-ordered-products in the free theory, we can again conclude that the $\boldsymbol{O}_{n}$ must have the form stated below eq. (52), and that, in particular, they are independent of the particular choice of $\theta$ so long as the support of $f$ is contained in the region where $\theta$ is equal to 1 . If we finally define $\boldsymbol{\delta}_{\theta L_{1}}(f \Phi)$ as in eq. (55) and set $Z=1+\delta Z$, then we can recast eq. (57) into the following form:

$$
\widetilde{\mathcal{S}}_{\theta L_{1}}(f \Phi)=\mathcal{S}_{\theta\left(L_{1}+\delta L_{1}\right)+\sum h_{j} \Lambda_{j}}\left(Z f \Phi+\boldsymbol{\delta}_{L_{1}}(f \Phi)\right) .
$$

\footnotetext{
${ }^{10}$ We will give a systematic analysis elsewhere of conditions that can be imposed on Wick powers and time-ordered-products involving derivatives.
} 
On $J(V)=J^{+}(V) \cup J^{-}(V)$ (the union of causal future and causal past of $V$ ), we decompose $h_{j}=h_{j-}+h_{j+}$, where $h_{j \pm}$ has compact support which does not intersect $J^{\mp}(V)$. If we now set

$$
W(\theta)=\mathcal{S}_{\theta\left(L_{1}+\delta L_{1}\right)}\left(\sum h_{j-} \boldsymbol{\Lambda}_{j}\right) .
$$

then we obtain by [4, thm. 8.1],

$$
\widetilde{\mathcal{S}}_{\theta L_{1}}(f \Phi)=W(\theta) \star \mathcal{S}_{\theta\left(L_{1}+\delta L_{1}\right)}\left(Z f \Phi+\boldsymbol{\delta}_{L_{1}}(f \Phi)\right) \star W(\theta)^{-1},
$$

which holds for all $f \in \mathcal{D}_{1}(M)$ with compact support in $V$. More generally, an analogous formula will hold if the expression $f \Phi$ is replaced by a sum of the form $\sum \alpha_{i} f_{i} \Phi_{i}$, where each $f_{i}$ has compact support in $V$.

We now obtain the desired formula eq. (52) from eq. (68) by removing the cutoff $\theta$ in the same way as in our definition of the interacting field in section 3.1: We consider a sequence of cutoff functions $\theta_{n}$ which are equal to 1 on globally hyperbolic open regions $V_{n}$ with compact closure that exhaust $M$. The interacting fields $T_{L_{1}+\delta L_{1}}\left(\prod \Phi_{i}\right)$ are then given in terms of the corresponding fields with cutoff interaction $\theta_{n}\left(\boldsymbol{L}_{1}+\delta \boldsymbol{L}_{1}\right)$ via eq. (42), and the interacting fields $\tilde{T}_{L_{1}}\left(\prod \tilde{\Phi}_{i}\right)$ are likewise given in terms of the corresponding fields with cutoff interaction $\theta_{n} \boldsymbol{L}_{1}$ by the tilde version of eq. (42). Using that the interacting fields with cutoff $\theta_{n}$ are related via the unitary $W\left(\theta_{n}\right)$ (see eq. (68)), one can now easily obtain a ${ }^{*}$-isomorphism $r: \tilde{\mathcal{B}}_{L_{1}}(M, \mathbf{g}) \rightarrow \mathcal{B}_{L_{1}+\delta L_{1}}(M, \mathbf{g})$ satisfying

$$
r\left(\widetilde{\mathcal{S}}_{L_{1}}(f \Phi)\right)=\mathcal{S}_{L_{1}+\delta L_{1}}\left(Z f \Phi+\boldsymbol{\delta}_{L_{1}}(f \Phi)\right),
$$

where $f$ is now an arbitrary test density of compact support. We can replace $f \Phi$ in the above formula by a sum $\sum \alpha_{i} f_{i} \Phi_{i}$ and differentiate the formula $n$ times with respect to to the parameters $\alpha_{i}$ (setting these parameters to zero afterwards). This gives us the desired identity (52).

\section{The Renormalization Group in Curved Spacetime}

\subsection{Scaling of interacting fields}

As explained in the previous section, it is possible to give a perturbative construction of the interacting quantum field theory that defines the interacting field, its Wick products, and their time ordered products as local, covariant fields. The construction of this theory depends on a prescription for defining Wick powers and their time-ordered-products in the corresponding free theory. As also explained, the definition of these quantities involves some ambiguities, and consequently the definition of the interacting field theory is also ambiguous. Nevertheless we showed in the previous subsection that these ambiguities can be analyzed in much the same way as in the free theory. The result of this analysis was summarized in eq. (52). 
In the present section we want to investigate the behavior of the interacting field, its Wick powers, and their time-ordered-products in the interacting theory under a rescaling of the metric by a constant conformal factor $\lambda$. As explained in the introduction, this analysis corresponds to a definition of the renormalization group in curved spacetime.

For the Wick powers and time-ordered-products in the free theory, the scaling behavior was analyzed at the end of section 2 using the "scaling map", $\sigma_{\lambda}$, (introduced in eq. (28) above), which associates to every element of $\mathcal{W}\left(M, \lambda^{2} \mathbf{g}, p(\lambda)\right)$ a corresponding element of $\mathcal{W}(M, \mathbf{g}, p)$, where $p(\lambda)=\left(\lambda^{-2} m^{2}, \xi\right)$ are the rescaled coupling constants. Choose an arbitrary, but fixed, prescription for defining Wick powers and their time-ordered-products in the free theory that satisfy the axioms of [14] and [15]. Let $\lambda$ be an arbitrary, but fixed, positive real number, and let $\Phi$ be a Wick power with engineering dimension $d$. We define

$$
{ }^{\lambda} \Phi[M, \mathbf{g}, p](f)=\lambda^{d} \sigma_{\lambda}\left(\Phi\left[M, \lambda^{2} \mathbf{g}, p(\lambda)\right](f)\right),
$$

and we similarly define ${ }^{\lambda} T\left(\prod^{\lambda} \Phi_{i}\right)[M, \mathbf{g}, p]$. It follows immediately that ${ }^{\lambda} \Phi$ and ${ }^{\lambda} T\left(\prod^{\lambda} \Phi_{i}\right)$ provide prescriptions for defining Wick powers and their time-ordered-products that also satisfy all of the axioms of [14] and [15]. As we have already noted, it then follows that the relation of this new $\lambda$-dependent prescription to the original prescription is of the form given by eq. (30) (but without the factors of $\lambda^{-d_{T}}$ occurring on the right side of that equation).

In order to analyze the scaling behavior of the fields in the interacting theory defined by the interaction Lagrangian density $\boldsymbol{L}_{1}=\kappa \varphi^{4} \boldsymbol{\epsilon}$, we proceed as follows. Our new $\lambda$-dependent prescription, eq. (70), for defining Wick powers and their time-orderedproducts for the free field gives rise, via the construction of section 3.1, to a new $\lambda$ dependent prescription for the perturbative construction of the corresponding interacting fields, which we denote by ${ }^{\lambda} \Phi_{L_{1}}$ and ${ }^{\lambda} T_{L_{1}}\left(\prod^{\lambda} \Phi_{i}\right)$, respectively. These quantities span an algebra of interacting fields denoted by ${ }^{\lambda} \mathcal{B}_{L_{1}}(M, \mathbf{g})$. From the uniqueness result, eq. (52), for the interacting Wick powers and their time-ordered-products derived in the preceeding subsection we then immediately get, for each $\lambda>0$, a *isomorphism

$$
r_{\lambda}:{ }^{\lambda} \mathcal{B}_{L_{1}}(M, \mathbf{g}) \rightarrow \mathcal{B}_{L_{1}+\delta L_{1}(\lambda)}(M, \mathbf{g})
$$

Here, $\delta \boldsymbol{L}_{1}(\lambda)$ is the $\lambda$-dependent counterterm Lagrangian of the form (51), whose $\lambda$ dependent coupling parameters are given by formal power series in $\kappa$. The coefficients in these power series are polynomials in $\log \lambda$ whose degree increases with $n$; for example

$$
\delta m^{2}(\lambda)=\sum_{n=1}^{\infty} p_{n}(\log \lambda) \kappa^{n},
$$

where the $p_{n}$ 's are polynomialst'

\footnotetext{
${ }^{11}$ It is possible to derive inequalities for the maximum degree of the polynomials $p_{n}$ as a function of the order $n$ in perturbation theory.
} 
It is not difficult to see that the relation between ${ }^{\lambda} \Phi_{L_{1}}$ and $\Phi_{L_{1}}$ is simply

$$
{ }^{\lambda} \Phi_{L_{1}}[M, \mathbf{g}, p](f)=\lambda^{d} \sigma_{\lambda}\left(\Phi_{L_{1}}\left[M, \lambda^{2} \mathbf{g}, p(\lambda)\right](f)\right),
$$

where here we have again denoted by $\sigma_{\lambda}$ the obvious extension of $\sigma_{\lambda}$ from $\mathcal{W}$ to $\mathcal{X}$. A similar formula holds for the time-ordered-products of the interacting fields. Consequently, if we compose $\sigma_{\lambda}$ with $r_{\lambda}$ constructed above, we obtain a ${ }^{*}$-isomorphism $\mathcal{R}_{\lambda}=r_{\lambda} \circ \sigma_{\lambda}$

$$
\mathcal{R}_{\lambda}: \mathcal{B}_{L_{1}}\left(M, \lambda^{2} \mathbf{g}, p(\lambda)\right) \rightarrow \mathcal{B}_{L_{1}+\delta L_{1}(\lambda)}(M, \mathbf{g}, p),
$$

where we indicate explicitly the dependence on the parameters $p$ in the free theory. Since the scaling map $\sigma_{\lambda}$ in the free theory satisfies $\sigma_{\lambda} \circ \sigma_{\lambda^{\prime}}=\sigma_{\lambda \lambda^{\prime}}$, it follows that

$$
\mathcal{R}_{\lambda} \circ \mathcal{R}_{\lambda^{\prime}}=\mathcal{R}_{\lambda \lambda^{\prime}}
$$

Using eq. (52) we find that the action of $\mathcal{R}_{\lambda}$ on an interacting time-ordered-product in the algebra $\mathcal{B}_{L_{1}}\left(M, \lambda^{2} \mathbf{g}, p(\lambda)\right)$ is given by

$$
\begin{gathered}
\mathcal{R}_{\lambda}\left(T_{L_{1}}\left(\prod_{i=1}^{n} \Phi_{i}\left(f_{i}\right)\right)\right)=\lambda^{-d_{T}} T_{L_{1}+\delta L_{1}(\lambda)}\left(\prod_{i=1}^{n} Z_{i}(\lambda) \Phi_{i}\left(f_{i}\right)\right)+ \\
\lambda^{-d_{T}} \sum_{P} T_{L_{1}+\delta L_{1}(\lambda)}\left(\prod_{I \in P} \boldsymbol{O}_{|I|}\left(\lambda ; \times_{i \in I} f_{i} \Phi_{i}\right) \prod_{j \notin I \forall I \in P} Z_{j}(\lambda) \Phi_{j}\left(f_{j}\right)\right) .
\end{gathered}
$$

Here, the $\lambda$-dependent field strength renormalization factors, $Z_{i}(\lambda)$, can be written as $Z_{i}(\lambda)=1+\sum_{n \geq 1} z_{i, n}(\log \lambda) \kappa^{n}$, where the coefficients $z_{i, n}$ depend at most polynomially on $\log \lambda$. The terms $\boldsymbol{O}_{n}\left(\lambda ; \times_{i} f_{i} \Phi_{i}\right)$ have the same form as eq. (52), and each of the terms in the sum on the right side of this equation is a formal power series in $\kappa$, whose coefficients are geometric tensors times polynomials in $\log \lambda$. For the special case of the interacting field $\varphi_{L_{1}}$, the above formula simplifies to

$$
\mathcal{R}_{\lambda}\left(\varphi_{L_{1}}(f)\right)=\lambda^{-1} Z(\lambda) \varphi_{L_{1}+\delta L_{1}(\lambda)}(f) .
$$

Equation (76) is our desired formula for the scaling behavior of the fields in the interacting quantum field theory. Although eq. (76) has many obvious similarities to the corresponding formula eq. (30) in the free theory, it should be noted that there are a number of important differences, in parallel with the differences in the general renormalization ambiguities of the free and interacting fields (see section 3.2 above). Most prominently, in the free field theory, the scaling relations (30) relate rescaled time-ordered-products to the unscaled time-ordered-products defined with respect to the "same Lagrangian", whereas 
the scaling relations eq. $(\sqrt{76})$ in the interacting theory $\left[{ }^{[2]}\right.$ relate the rescaled time-orderedproducts for the interaction Lagrangian $\boldsymbol{L}_{1}$, to the unscaled time-ordered-products defined with respect to the interaction Lagrangian $\boldsymbol{L}_{1}(\lambda)=\boldsymbol{L}_{1}+\delta \boldsymbol{L}_{1}(\lambda)$. Another important difference between the scaling relations (76) and (30) is the occurrence of the field strength renormalization factors, $Z_{i}(\lambda)$, in the interacting field theory, while such factors are absent in the free theory. As a consequence, the interacting fields do not in general have an almost homogeneous scaling behavior.

Given any fixed renormalization prescription, eq. (174) shows that the theory defined for the rescaled metric and rescaled parameters of the free theory is equivalent to the original theory with a modified Lagrangian $\delta \boldsymbol{L}_{1}$. The $\lambda$-dependence of the parameters $\delta m^{2}(\lambda), \delta z(\lambda), \delta \xi(\lambda)$, and $\delta \kappa(\lambda)$ in $\delta \boldsymbol{L}_{1}(\lambda)$ define the renormalization group flow of the theory. As already mentioned $\delta m^{2}, \delta z, \delta \xi$, and $\delta \kappa$ are formal power series in $\kappa$. These quantities also depend upon the parameters appearing in $\boldsymbol{L}_{0}$, so $\delta m^{2}, \delta z, \delta \xi$, and $\delta \kappa$ should be viewed as effectively being functions of $\kappa, m^{2}$, and $\xi$, as well as of $\lambda$. However, it should be noted that the renormalization group flow is independent of the spacetime metric $\mathrm{g}$.

The physical meaning of the renormalization group flow can perhaps be best explained by imagining that a quantum field theory textbook from an ancient civilization has been discovered. This textbook contains a complete description of perturbative renormalization theory for the scalar field (11) as well as complete instructions on how to build apparatuses to prepare states of the theory and to make measurements (see the discussion at the end of section 3.1). It also records the results of these measurements and compares them with theoretical predictions (to some appropriately high order in perturbation theory), thereby fixing the parameters of the theory. However, the one piece of information that is missing is the system of units used by the ancient civilization; in other words, the lengthscale, $l$, used by the ancient civilization to define the fundamental unit of length (in terms of which other units, such as mass, are defined in the standard way) is not presently known. This lengthscale enters both the renormalization prescription given in the book (since, the specification of a particular locally constructed Hadamard parametrix and the renormalization prescription for defining time-ordered-products require a specification of a unit of length) as well as the instructions for building the apparatuses and making the measurements. Suppose, now, that a physicist from the present era tries to verify the experimental claims made in the book. He makes a guess, $l^{\prime}$, as to the value of $l$, which, as it turns out, differs from $l$ by a factor of $\lambda^{-1}$, i.e., $l^{\prime}=l / \lambda$. Since the present-day

\footnotetext{
${ }^{12}$ For the classical interacting field, the scaling relations also do not involve a modification of the interaction Lagrangian, as can be seen from the fact that the classical Lagrangian $\boldsymbol{L}$ (and the corresponding classical nonlinear equations of motion) is manifestly invariant under transformation $\mathbf{g} \rightarrow \lambda^{2} \mathbf{g}, \varphi \rightarrow \lambda^{-1} \varphi$, $m^{2} \rightarrow \lambda^{-2} m^{2}$ and $\xi \rightarrow \xi$. This can also be seen, more indirectly, in present formalism if one keeps explicitly the dependence of our constructions on $\hbar$, so that the corresponding classical theory corresponds to the limit $\hbar \rightarrow 0$. This is most naturally done by introducing $\hbar$ as an explicit parameter in our definition of the product " $\star$ ", eq. (6), in our algebra $\mathcal{W}$ (and likewise $\mathcal{X}$ ), see $[\mathbb{8}]$.
} 
physicist will normalize the spacetime metric so that a rod of length $l^{\prime}$ will have unit length (whereas the ancient civilization assumed that a rod of length $l$ has unit length), the spacetime metric $\mathbf{g}^{\prime}$ used by the present-day physicist will differ from the metric $\mathbf{g}$ that would have been used by the ancient civilization by $\mathbf{g}^{\prime}=\lambda^{2} \mathbf{g}$. Correspondingly, all of the experimental apparatuses built by the present-day physicist will be a factor of $\lambda$ smaller in all linear dimensions than intended by the author of the ancient textbook. When the present day physicist completes his experiments, he will find that his results disagree with the results reported in the book. He will find that this disagreement will be alleviated he compares his results to the theoretical predictions obtained from the renormalization prescription given in the book by using the mass parameter $m^{\prime}=\lambda^{-1} m$ in $\boldsymbol{L}_{0}$ rather than $m$, but disagreements will still remain. However, if, in addition to the substitution $m^{\prime}=\lambda^{-1} m$ in $\boldsymbol{L}_{0}$, the present-day physicist also modifies the interaction Lagrangian $\boldsymbol{L}_{1}$ by eq. (51) (with $\delta z, \delta m^{2}$, etc. given by eq. (72)), then he will find exact agreement with the theoretical predictions obtained from the renormalization prescription given in the book, provided that he also redefines the field variables in accordance with the ${ }^{*}$-isomorphism $\mathcal{R}_{\lambda}$ given by eq. (76). In other words, when the properties of the scalar field are investigated on a scale different from that used by the ancient civilization, its properties will be found to differ by a "running of coupling constants" in the interaction Lagrangian.

The quantity $\delta \kappa(\lambda)$ can be viewed as modifying the nonlinear coupling parameter $\kappa$ appearing in the original interaction Lagrangian $\boldsymbol{L}_{1}$. However, it should be noted that the quantities $\delta m^{2}(\lambda), \delta z(\lambda)$, and $\delta \xi(\lambda)$ all correspond to parameters appearing in the original free Lagrangian, $\boldsymbol{L}_{0}$, rather than $\boldsymbol{L}_{1}$. It would be natural to try to interpret these terms in $\boldsymbol{L}_{1}$ as corresponding to changes in the coupling constants $m^{2}, z=1$, and $\xi$ appearing in $\boldsymbol{L}_{0}$. However, we do not know how to justify such an interpretation because we have only constructed the interacting theory at the level of a formal perturbation expansion. Therefore, we cannot compare an interacting theory based on the free Lagrangian $\boldsymbol{L}_{0}$ with an interacting theory based on the free Lagrangian $\boldsymbol{L}_{0}+\delta \boldsymbol{L}_{0}$, where $\delta \boldsymbol{L}_{0}=\frac{1}{2}\left[\delta z(\nabla \varphi)^{2}+\right.$ $\left.\delta \xi R \varphi^{2}+\delta m^{2} \varphi^{2}\right] \epsilon$.

Finally, as we have already noted, the renormalization group flow occurs in the parameter space of the theory and is independent of the spacetime metric. Thus, in order to calculate (or measure) the renormalization group flow, it suffices to restrict attention to a single spacetime, provided that the spacetime is not so special that possible curvature couplings do not occur. Thus, for example, in the theory with Lagrangian (1), the only coupling to curvature occurs in the term $\xi R \varphi^{2}$, so it would suffice to calculate the renormalization group flow in any spacetime with nonvanishing scalar curvature. We will indicate how to calculate renormalization group flow in curved spacetime in terms of Feynman diagrams in appendix B. However, we point out here that a great deal of information about the renormalization group flow can be deduced from dimensional 
considerations as well as from some simple properties that hold in special spacetimest ${ }^{[3}$. From dimensional considerations alone, it follows that the dependence of $\delta m^{2}(\lambda), \delta z(\lambda)$, $\delta \xi(\lambda), \delta \kappa(\lambda)$ on the parameters $m^{2}, \xi$, and $\kappa$ must be of the form $\delta m^{2}(\lambda)=m^{2} F_{m^{2}}(\lambda, \xi, \kappa)$, $\delta z(\lambda)=F_{z}(\lambda, \xi, \kappa), \delta \xi(\lambda)=F_{\xi}(\lambda, \xi, \kappa), \delta \kappa(\lambda)=F_{\kappa}(\lambda, \xi, \kappa)$. However, it is possible (and would be very natural) to choose a prescription for defining free field Wick products and their time-ordered-products in an arbitrary spacetime such that in the special case of Minkowski spacetime, this prescription does not depend upon the irrelvant parameter $\xi$. It follows immediately that with such a renormalization prescription, the renormalization group flow cannot depend upon $\xi$ in Minkowski spacetime and, therefore - since the flow is independent of the spacetime metric - the flow cannot depend upon $\xi$ in any spacetime. More generally, it is possible (and would be very natural) to choose a prescription for defining free field Wick products and their time-ordered-products in an arbitrary spacetime such that in the special case of a spacetime with constant scalar curvature $R$ (such as deSitter spacetime), the only dependence of the prescription on the parameters $m^{2}$ and $\xi$ occurs in the combination $m^{2}+\xi R$. This condition implies that (in all spacetimes), the renomalization group flow must take the form

$$
\begin{aligned}
\delta m^{2} & =m^{2} G_{1}(\lambda, \kappa) \\
\delta \xi & =\xi G_{1}(\lambda, \kappa)+G_{2}(\lambda, \kappa) \\
\delta z & =G_{3}(\lambda, \kappa) \\
\delta \kappa & =G_{4}(\lambda, \kappa)
\end{aligned}
$$

The functions $G_{1}, G_{3}$, and $G_{4}$ can all be determined by calculations done entirely in Minkowski spacetime; the function $G_{2}$ cannot be determined by calculations in Minkowski spacetime but could be determined by calculations done, e.g., in deSitter spacetime.

\subsection{Fixed points, essential vs. inessential coupling parameters}

In the previous section we have seen that a rescaling of the spacetime metric by a constant conformal factor, $\mathbf{g} \rightarrow \lambda^{2} \mathbf{g}$, (a "change of length scale") gives rise to different definitions of the interacting field theory. The relation between the definitions of the field theory at different length scales is given by the renormalization group. It is of interest to ask at what points in the parameter space of the theory the definition of a field theory is actually "independent" of the scale at which it is defined. Such points are usually referred to as "fixed points".

Naively, one might attempt to define a fixed point as a point in parameter space at which the $\lambda$-derivatives of $\delta m^{2}(\lambda), \delta z(\lambda), \delta \xi(\lambda)$, and $\delta \kappa(\lambda)$ all vanish. However, this definition would be too restrictive because it excludes points where the renormalization

\footnotetext{
${ }^{13}$ We are indebted to K.-H. Rehren, C.J. Fewster, and K. Fredenhagen for bringing this point to our attention.
} 
group flow is nonvanishing but corresponds merely to a redefinition of field variables. One would like to define the notion of fixed points so that it also includes points in parameter space where the renormalization group flow is nonvanishing but is tangent to a trivial flow corresponding to a field redefinition.

To see more explicitly the nature of such trivial flows, consider a field theory with Lagrangian $\boldsymbol{L}(\varphi)$ and consider a mapping $\varphi \rightarrow F(\varphi)$ on field space such that $F(\varphi)(x)$ depends only on $\varphi(x)$ and finitely many of its covariant derivatives at the point $x$. Then, although the Lagrangian $\boldsymbol{L}(\varphi)$ and $\boldsymbol{L}(F(\varphi)$ ) may look very different (i.e., different kinds of couplings and different values of coupling parameters), they nevertheless would define an equivalent classical field theory. Thus, at the classical level, there is a wide class of trivial flows in parameter space that correspond to field redefinitions. However, the situation is far more restrictive for a field with Lagrangian (11) if we want the field redefinition to keep the Lagrangian in a perturbatively renormalizable form. It is not difficult to see that (in 4 dimensions) this leaves us only with the possibility to multiply the field by a constant, i.e., the only possible form of $F$ is $F(\varphi)=s \varphi$. The new classical Lagrangian $\boldsymbol{L}(s) \equiv \boldsymbol{L}(F(\varphi))$ is then

$$
\boldsymbol{L}(s)=\frac{1}{2}\left[s^{2}(\nabla \varphi)^{2}+s^{2}\left(m^{2}+\xi R\right) \varphi^{2}+s^{4} \kappa \varphi^{4}\right] \boldsymbol{\epsilon}
$$

If one splits this Lagrangian into its free and interacting parts via $\boldsymbol{L}(s)=\boldsymbol{L}_{0}+\boldsymbol{L}_{1}(s)$ with $\boldsymbol{L}_{0}=\frac{1}{2}\left[(\nabla \varphi)^{2}+m^{2} \varphi^{2}+\xi R \varphi^{2}\right] \boldsymbol{\epsilon}$, the interaction Lagrangian takes the form

$$
\boldsymbol{L}_{1}(s)=\frac{1}{2}\left[\left(s^{2}-1\right)(\nabla \varphi)^{2}+\left(s^{2}-1\right)\left(m^{2}+\xi R\right) \varphi^{2}+s^{4} \kappa \varphi^{4}\right] \boldsymbol{\epsilon} .
$$

Therefore, one might expect that the "one-parameter flow" defined by eq. (80) — with $s$ taken to be an arbitrary power series in $\kappa$-would correspond to a trivial flow in the parameter space of the theory in the sense that the theory constructed from the interaction Lagrangian $\boldsymbol{L}_{1}(s)$ would be equivalent to the theory constructed from the original interaction Lagrangian $\boldsymbol{L}_{1}=\frac{1}{2} \kappa \varphi^{4} \boldsymbol{\epsilon}$.

However, the actual situation is somewhat more complicated than the above considerations might suggest. The theories constructed from the interaction Lagrangians $\boldsymbol{L}_{1}(s)$ and $\boldsymbol{L}_{1}$ will depend upon the specific choice of renormalization prescription, and, for any given prescription, we see no reason why these two theories need be equivalent. Indeed, it appears far from clear that there exists any renormalization prescription that gives equivalence of the two theories. Nevertheless, we shall now show that, for any fixed renormalization prescription, there exists some one-parameter family of interaction Lagrangians, $\boldsymbol{K}_{1}(s)$, such that the theories constructed from $\boldsymbol{K}_{1}(s)$ are equivalent to the theory constructed from $\boldsymbol{L}_{1}$ in the sense that the algebras $\mathcal{B}_{K_{1}(s)}(M, \mathbf{g})$ and $\mathcal{B}_{L_{1}}(M, \mathbf{g})$ are isomorphic. Furthermore, the action of this isomorphism on the interacting field corresponds to the simple field redefinition $F(\varphi)=N(s) \varphi$, where $N(s)$ is a formal power series with the propery $N(s=1)=1$. The precise statement of this result is as follows: 
Theorem 4.1. Let $s=1+\sum_{i \geq 1} s_{i} \kappa^{i}$ be a formal power series in $\kappa$ with real coefficients. Then there exists an interaction Lagrangian $\boldsymbol{K}_{1}(s)$ of the same form as the original Lagrangian, a formal power series $N(s)$ and a ${ }^{*}$-isomorphism $\rho_{s}: \mathcal{B}_{L_{1}}(M, \mathbf{g}) \rightarrow \mathcal{B}_{K_{1}(s)}(M, \mathbf{g})$ such that

$$
\rho_{s}\left[\varphi_{L_{1}}(f)\right]=N(s) \varphi_{K_{1}(s)}(f)
$$

for all $f \in \mathcal{D}_{1}(M)$, and such that $N(s=1)=1$ and $\boldsymbol{K}_{1}(s=1)=\boldsymbol{L}_{1}$.

A proof of this theorem is given in appendix A.

According to the above theorem, it is natural to view the interaction Lagrangians $\boldsymbol{L}_{1}$ and $\boldsymbol{K}_{1}(s)$ as defining the same quantum field theories and $\rho_{s}$ as implementing the field redefinition. If we choose coordinates on the space of parameters in the Lagrangian so that the coordinate vector field of one of the coordinates is tangent to the flow defined by $\boldsymbol{K}_{1}(s)$, then we refer to this coordinate as an inessential parameter of the theory (see, e.g., [23]). We define a fixed point of the renormalization group flow to be a point at which only the inessential parameter changes under the flow. More precisely, if $\lambda \rightarrow \boldsymbol{L}_{1}(\lambda)$ is the renormalization group flow, then we say that we are at a fixed point if there is a 1-parameter family $\lambda \rightarrow s(\lambda)$ such that

$$
\boldsymbol{L}_{1}(\lambda)=\boldsymbol{K}_{1}(s(\lambda)) \text { for all } \lambda>0 .
$$

This relation can be differentiated with respect to $\log \lambda$, thereby relating a fixed point to a zero of a suitably defined $\beta$-function. For this, we write $\boldsymbol{L}_{1}(\lambda)=\boldsymbol{L}_{1}+\delta \boldsymbol{L}_{1}(\lambda)$, and $\boldsymbol{K}_{1}(s)=\boldsymbol{L}_{1}+\delta \boldsymbol{K}_{1}(s)$, and we denote the parameters in $\delta \boldsymbol{L}_{1}(\lambda)$ by $\delta z(\lambda), \delta \kappa(\lambda)$ etc. and the parameters in $\delta \boldsymbol{K}_{1}(s)$ by $\delta \tilde{z}(s), \delta \tilde{\kappa}(s)$ etc. We define

$$
\beta_{\kappa} \equiv \frac{\partial}{\partial \log \lambda} \delta \kappa-\left.\frac{\partial}{\partial s} \delta \tilde{\kappa}\left(\frac{\partial}{\partial s} \delta \tilde{z}\right)^{-1} \frac{\partial}{\partial \log \lambda} \delta z\right|_{\lambda=s=1} .
$$

Then a fixed point beta functions $\left.\beta_{m^{2}}, \beta_{\xi}\right)$.

Acknowlegements: This work was supported in part by NSF grant PHY00-90138 to the University of Chicago. Part of this research was carried out during the program on Quantum Field Theory in Curved Spacetime at the Erwin Schrödinger Institute, and we wish to thank the Erwin Schrödinger Institute for its hospitality.

\footnotetext{
${ }^{14}$ If $\boldsymbol{K}_{1}(s)$ were actually of the form (80), then the $\beta$-function for $\kappa$ would be given by $\beta_{\kappa} \equiv$ $\left.\frac{\partial}{\partial \log \lambda}(\delta \kappa(\lambda)-2 \kappa \delta z(\lambda))\right|_{\lambda=1}$

${ }^{15}$ It should be noted that the interacting theory has been constructed only at the level of a formal perturbation expansion, it will not be possible to reliably determine fixed points unless they occur near $\kappa=0$.
} 


\section{A Appendix A}

In this appendix we give a proof of theorem 4.1. Mainly for notational simplicity, we will assume throughout this proof that $\xi=m^{2}=0$, so that $\boldsymbol{L}_{0}=\frac{1}{2}(\nabla \varphi)^{2} \boldsymbol{\epsilon}$; the general case can be treated in exactly the same way. Consider the Lagrangian density $\delta \boldsymbol{L}_{0}=$ $\frac{1}{2} \delta s(\nabla \varphi)^{2} \boldsymbol{\epsilon}$ with $\delta s=s^{2}-1$, and a cutoff function $\theta$ which is equal to 1 in a neighborhood of the closure $\bar{V}$ of a globally hyperbolic neighborhood $V$ with compact closure and with a Cauchy surface of the form $\Sigma \cap V$, where $\Sigma$ is a Cauchy surface for $M$. Although $\delta \boldsymbol{L}_{0}$ is, of course, only quadratic in the field $\varphi$, we may consider it as an "interaction Lagrangian," and we can define, by eqs. (35) respectively (36) (with $\boldsymbol{L}_{1}$ in those equations replaced by $\delta \boldsymbol{L}_{0}$ ), the corresponding "interacting" fields as formal power series in $\delta s$ (or, more properly, as formal power series in $\kappa$, since $s$ itself is a formal power series in $\kappa$ ).

The first step in our proof is to show that the "interacting fields" $\varphi_{\theta \delta L_{0}}(f)$ with $f$ a smooth test density of compact support in $V$ satisfy exactly the same algebraic relations as the fields $s^{-1} \varphi(f)$. Furthermore, we show that the "interacting time-ordered-products" $T_{\theta \delta L_{0}}\left(\prod \Phi_{i}\left(f_{i}\right)\right.$ ) (with the support of $f_{i}$ contained in $V$ ) satisfy commutation relations with the field $\varphi_{\theta \delta L_{0}}(f)$ that have exactly the same form as the commutation relations of $s^{-N} T\left(\prod \Phi_{i}\left(f_{i}\right)\right)$ with $s^{-1} \varphi(f)$ given in [15], where $N$ is the number of free field factors in the time-ordered-product. We formulate this result as a lemma.

Lemma A.1. For all smooth test densities with support in $V$, we have that

$$
\varphi_{\theta \delta L_{0}}\left(\nabla^{a} \nabla_{a} f\right)=0, \quad \varphi_{\theta \delta L_{0}}(f)^{*}=\varphi_{\theta \delta L_{0}}(\bar{f}), \quad\left[\varphi_{\theta \delta L_{0}}\left(f_{1}\right), \varphi_{\theta \delta L_{0}}\left(f_{2}\right)\right]=i s^{-2} \Delta\left(f_{1}, f_{2}\right) \mathbb{1}
$$

in the sense of formal power series ${ }^{\text {Tb }}$ in $\kappa$. More generally it holds that

$$
\begin{aligned}
& {\left[T_{\theta \delta L_{0}}\left(\prod_{i=1}^{n} \Phi_{i}\left(f_{i}\right)\right), \varphi_{\theta \delta L_{0}}\left(f_{n+1}\right)\right]=} \\
& s^{-2} \sum_{j=1}^{n} T_{\theta \delta L_{0}}\left(\Phi_{1}\left(f_{1}\right) \ldots i \sum_{(a)} \frac{\partial \Phi_{j}}{\partial \nabla_{(a)} \varphi}\left(f_{n+1} \Delta_{(a)} f_{j}\right) \ldots \Phi_{n}\left(f_{n}\right)\right),
\end{aligned}
$$

where $f_{n+1} \Delta_{(a)} f_{j}$ was defined in eq. (22).

Proof. In order to prove the first relation in eq. (84), we first expand

$$
\varphi_{\theta \delta L_{0}}(f)=\varphi(f)+\sum_{n \geq 1} \frac{(i \delta s)^{n}}{n !} R(f \varphi ; \underbrace{\theta \boldsymbol{L}_{0}, \ldots, \theta \boldsymbol{L}_{0}}_{n \text { factors }}) .
$$

\footnotetext{
${ }^{16}$ For example, $s^{-1}$ is defined as the formal power series $\sum_{n}(-1)^{n}\left(\sum_{i \geq 1} s_{i} \kappa^{i}\right)^{n}$.
} 
Since $\boldsymbol{L}_{0}$ is only quadratic in the field $\varphi$, the totally retarded products (86) can be given in closed form in terms of the retarded Green's function $\Delta_{r e t}$ for $\nabla^{a} \nabla_{a}$,

$$
\begin{aligned}
R\left(\varphi(x) ; \prod_{i=1}^{n} \boldsymbol{L}_{0}\left(y_{i}\right)\right)=i^{n} \sum_{i_{1} \cdots i_{n}} \Delta_{r e t}\left(x, y_{i_{1}}\right) \overleftarrow{\nabla} \vec{\nabla} \times \\
\left.\Delta_{r e t}\left(y_{i_{1}}, y_{i_{2}}\right) \overleftarrow{\nabla} \vec{\nabla} \cdots \Delta_{r e t}\left(y_{i_{n-1}}\right), y_{i_{n}}\right) \overleftarrow{\nabla} \vec{\nabla} \varphi\left(y_{i_{n}}\right)
\end{aligned}
$$

where the summation over the spacetime index has been suppressed in the expression $\overleftarrow{\nabla} \vec{\nabla}$. We now use this expression to analyze the oparator $R\left(\nabla^{a} \nabla_{a} f \varphi ; \times^{n} \theta \delta \boldsymbol{L}_{0}\right)$, where $f$ is a test density supported in $V$. In order to do this, we perform the following steps: We use $\nabla^{a} \nabla_{a} \Delta_{\text {ret }}=\delta$ to turn the first retarded Green's function on the right side of eq. (87) into a delta-function. We then use that $\theta$ is 1 in $V$ and that $f$ has support in $V$ and perform $n$ successive partial integrations in order to turn the $\overleftarrow{\nabla} \vec{\nabla}$ derivatives into $\vec{\nabla} \vec{\nabla}$ derivatives which will now hit a single retarded Green's function, thus resulting each time in a new delta-function. If this is done, then one obtains $R\left(\nabla^{a} \nabla_{a} f \varphi ; \times^{n} \theta \delta \boldsymbol{L}_{0}\right)=0$, thereby proving the first equation in (84). The second equation in (84) follows from the unitarity of the relative $S$-matrix $\mathcal{S}_{\theta \delta L_{0}}(f \varphi)$ for real-valued $f$.

We will demonstrate eq. (85) in the case of Wick powers of the form $\varphi^{k}$; Wick powers with derivatives and time-ordered-products can be treated similarly. The proof of the last relation in eq. (84) is included as the special case $k=1$. Our starting point is the relation [8][]

$$
\begin{aligned}
{\left[\varphi_{\theta \delta L_{0}}^{k}\left(x_{1}\right), \varphi_{\theta \delta L_{0}}\left(x_{2}\right)\right] } & =\sum_{n \geq 0} \frac{(i \delta s)^{n}}{n !} \int_{M^{\times n}} \prod_{j} \theta\left(y_{j}\right) \times \\
& \left(R\left(\varphi^{k}\left(x_{1}\right) ; \varphi\left(x_{2}\right) \prod_{j=1}^{n} \boldsymbol{L}_{0}\left(y_{j}\right)\right)-R\left(\varphi\left(x_{2}\right) ; \varphi^{k}\left(x_{1}\right) \prod_{j=1}^{n} \boldsymbol{L}_{0}\left(y_{j}\right)\right)\right),
\end{aligned}
$$

where the integral is over the " $y$ "-variables. We will now simplify the terms under the sum in the above equation, starting with the terms $R\left(\varphi^{k}\left(x_{1}\right) ; \varphi\left(x_{2}\right) \prod_{j=1}^{n} \boldsymbol{L}_{0}\left(y_{j}\right)\right)$. For this, we use the fact that the time ordered products with a factor $\varphi$ can be shown to satisfy the following requirement in addition to any other requirements imposed so far $\$$ :

$$
\left(\nabla^{a} \nabla_{a}\right)_{x} T\left(\varphi(x) \prod_{j=1}^{n} \Phi_{j}\left(y_{j}\right)\right)=i \sum_{j=1}^{n} \sum_{(b)} \nabla_{(b)} \delta\left(y_{j}, x\right) T\left(\Phi_{1}\left(y_{1}\right) \cdots \frac{\partial \Phi_{j}}{\partial \nabla_{(b)} \varphi}\left(y_{j}\right) \cdots \Phi_{n}\left(y_{n}\right)\right)
$$

\footnotetext{
${ }^{17} \mathrm{~A}$ general formula of this kind which holds within the LSZ-framework in Minkowski spacetime was first given by [11].

${ }^{18} \mathrm{~A}$ proof of this equation for Minkowski spacetime appears in [9]. This proof can be generalized to curved spacetimes by suitably modifying the constructions of time ordered products given in [15].
} 
for all fields $\Phi_{j}$. It can be seen that this implies

$$
\begin{array}{r}
R\left(\varphi^{k}\left(x_{1}\right) ; \varphi\left(x_{2}\right) \prod_{j=1}^{n} \boldsymbol{L}_{0}\left(y_{j}\right)\right)=i \sum_{l=1}^{n} \nabla_{a} \Delta_{r e t}\left(y_{l}, x_{2}\right) R\left(\varphi^{k}\left(x_{1}\right) ; \nabla^{a} \varphi\left(y_{l}\right) \prod_{j \neq l} \boldsymbol{L}_{0}\left(y_{j}\right)\right) \\
+i \Delta_{r e t}\left(x_{1}, x_{2}\right) R\left(\frac{\partial \varphi^{k}}{\partial \varphi}\left(x_{2}\right) ; \prod_{j=1}^{n} \boldsymbol{L}_{0}\left(y_{j}\right)\right)
\end{array}
$$

for the retarded products appearing in eq. (88). Now the retarded products in the sum on the right side of eq. (90) again contain a factor $\varphi$, and we can a similar argument as above to further simplyfy each of these terms. Repeating this procedure $n$ times, we can rewrite the right side of eq. (90) as

$$
\begin{aligned}
=i \sum_{N=0}^{n} i^{N} \sum_{l_{1} \cdots l_{N}} \Delta_{r e t}\left(x_{1}, y_{l_{1}}\right) \overleftarrow{\nabla} \vec{\nabla} \Delta_{r e t}\left(y_{l_{1}}, y_{l_{2}}\right) \overleftarrow{\nabla} \vec{\nabla} \cdots \Delta_{r e t}\left(y_{l_{N}}, x_{2}\right) & \\
& \times R\left(\frac{\partial \varphi^{k}}{\partial \varphi}\left(x_{2}\right) ; \prod_{j \neq l_{1}, \ldots, l_{N}} \boldsymbol{L}_{0}\left(y_{j}\right)\right)
\end{aligned}
$$

The second term $R\left(\varphi\left(x_{2}\right) ; \varphi^{k}\left(x_{1}\right) \prod_{j=1}^{n} \boldsymbol{L}_{0}\left(y_{j}\right)\right)$ under the sum in eq. (88) can be written in the form of expression (91) with $x_{1}$ and $x_{2}$ exchanged. We now substitute these expressions back into (88) and perform the following steps: We use that $x_{1}, x_{2} \in V$, that $\theta \equiv 1$ on $V$ and the support property $\operatorname{supp} \Delta_{\text {ret }} \subset\left\{\left(x_{1}, x_{2}\right) \in M \times M \mid x_{1} \in J^{+}\left(x_{2}\right)\right\}$ to bring each of turn each of the $\overleftarrow{\nabla} \vec{\nabla}$ derivatives on the variables $y_{l_{j}}$ into a $\vec{\nabla} \vec{\nabla}$ derivative acting on a single retarded Green's function via a partial integration. We then use that $\nabla^{a} \nabla_{a} \Delta_{\text {ret }}=\delta$ and use these delta-functions to get rid of the string of retarded Green's functions in (91). We now exploit the relation $\Delta_{\text {ret }}\left(x_{1}, x_{2}\right)=\Delta_{a d v}\left(x_{2}, x_{1}\right)$ (with $\Delta_{a d v}$ the advanced Green's function), as well as $\Delta=\Delta_{a d v}-\Delta_{\text {ret }}$, which enables one to get rid of all retarded Green's functions in favor of commutator functions. We finally collect similar terms and use the geometric series $\sum_{N=0}^{\infty}(\delta s)^{N}=s^{-2}$ (here it must be used that $s$ has the special form $1+\sum_{i \geq 1} s_{i} \kappa^{i}$, or else the formal power series $s^{-2}$ is not well-defined). If all this is done, then one obtains (85) for the special case of a Wick product of the form $\varphi^{k}$.

It follows from eqs. (84) and (85) that the linear map

$$
\rho_{\theta}\left[\varphi\left(f_{1}\right) \star \cdots \star \varphi\left(f_{n}\right)\right] \equiv s^{n} \varphi_{\theta \delta L_{0}}\left(f_{1}\right) \star \cdots \star \varphi_{\theta \delta L_{0}}\left(f_{n}\right)
$$

defines a *-homomorphism from the canonical commutation relation algebra $\mathcal{A}(V, \mathbf{g})$ into the subalgebra of $\mathcal{X}(M, \mathbf{g})$ spanned by products of the fields $\varphi_{\theta \delta L_{0}}(f)$, where $f$ is an arbitrary test density supported in $V$. Since the algebra $\mathcal{A}(V, \mathbf{g})$ is simple, $\rho_{\theta}$ is injective. 
It is possible to see that the homomorphism $\rho_{\theta}$ can be extended by continuity unique ${ }^{*}$-homorphism from $\mathcal{W}(V, \mathbf{g})$, (and therefore also from $\left.\mathcal{X}(V, \mathbf{g})\right)$ to $\mathcal{X}(M, \mathbf{g})$. We will denote this extension by the same symbol $\rho_{\theta}$.

We will now construct for any set of test densities $f_{i}$ of compact support in $V$ and for any set of fields $\Phi_{i} \in \mathcal{V}$ an element $F\left(s ; \times_{i} f_{i} \Phi_{i}\right) \in \mathcal{X}(V, \mathbf{g})$ such that

$$
\rho_{\theta}\left[F\left(s ; \times_{i=1}^{n} f_{i} \Phi_{i}\right)\right]=s^{N} T_{\theta \delta L_{0}}\left(\prod_{i=1}^{n} \Phi_{i}\left(f_{i}\right)\right),
$$

where $N$ is the number of factors of $\varphi$ in the time-ordered-product. Furthermore, we claim that quantities $F\left(s ; \times_{i} f_{i} \Phi_{i}\right)$ are independent of the particular choice of $\theta$ and $V$ and define in fact a new, $s$-dependent prescription for defining time-ordered-products in the free theory, i.e. that

$$
\tilde{T}\left(\prod_{i=1}^{n} \tilde{\Phi}_{i}\left(f_{i}\right)\right) \equiv F\left(s ; \times_{i=1}^{n} f_{i} \Phi_{i}\right)
$$

satisfies all the requirements of our uniqueness theorem for time-ordered-products in the free theory.

Before we sketch the proof of eq. (93) and the claims following that equation, we would like to mention that we see no reason obvious why the prescription $\tilde{T}$ should coincide with the original prescription $T$. As we will see below, the possible failure of $\tilde{T}$ to coincide with $T$ is the reason why the Lagrangian $\boldsymbol{K}_{1}(s)$ in the theorem need not have the simple form expected from the classical theory.

It follows from the relation

$$
\rho_{\theta^{\prime}}=\operatorname{Ad}\left(U\left(\theta^{\prime}, \theta\right)\right) \circ \rho_{\theta}
$$

(with $U\left(\theta, \theta^{\prime}\right)$ defined as in eq. (39), but with $\boldsymbol{L}_{1}$ in that equation replaced by $\delta \boldsymbol{L}_{0}$ ) that if elements $F\left(s ; \times_{i} f_{i} \Phi_{i}\right)$ satisfying eq. (93) exist, then they must be independent of $\theta$. We now explain how to construct these elements. By definition of $\rho_{\theta}$ given in eq. (92) we already know that eq. (93) holds for the field $s \varphi_{\theta \delta L_{0}}(f)$ with $F(s ; f \varphi)$ given by $\varphi(f)$ in that case. The construction of $F\left(s ; \times_{i} f_{i} \Phi_{i}\right)$ for a general time-ordered-product $s^{N} T_{\theta \delta L_{0}}\left(\prod \Phi_{i}\left(f_{i}\right)\right)$ is as follows: On the algebra $\mathcal{W}(M, \mathbf{g})$, we consider, for all $t_{i} \in \mathcal{E}^{\prime}(M, \mathbf{g})$, the (commutative, associative) producter

$$
\chi^{n} \mathcal{W}(M, \mathbf{g}) \rightarrow \mathcal{W}(M, \mathbf{g}), \quad \times_{i=1}^{n}\left[t_{i}\right] \rightarrow W\left(\times_{i=1}^{n}\left[t_{i}\right]\right) \equiv\left[t_{1} \otimes_{\text {sym }} \cdots \otimes_{\text {sym }} t_{n}\right]
$$

\footnotetext{
${ }^{19}$ It was shown in [14] that the Hörmander topology on the spaces $\mathcal{E}_{\text {sym }}^{\prime}\left(M^{\times n}\right)$ (see eq. (\$)) induces a natural topology on the algebra $\mathcal{W}(V, \mathbf{g})$ and likewise on the algebra $\mathcal{X}(M, \mathbf{g})$. It can then be seen that the map $\rho_{\theta}$ defined in eq. (92) is continuous with respect to this topology.

20 If the $t_{i}$ are given by smooth densities $f_{i}$ on $M$, then the product $W\left(\left[f_{1}\right], \ldots,\left[f_{n}\right]\right)$ corresponds to the normal ordered product $: \varphi\left(f_{1}\right) \cdots \varphi\left(f_{n}\right):_{\omega}$, where the normal ordering is done with respect to the quasifree state $\omega$ used in the definition of the algebra $\mathcal{W}$.
} 
We also denote by $W$ the corresponding product on $\mathcal{X}(M, \mathbf{g})$ when each $t_{i}$ is a formal power series in $\kappa$ with coefficients in $\mathcal{E}^{\prime}(M, \mathbf{g})$. Then it follows from the third equation in (84) that, within $V$, we have

$$
\left[W\left(\times_{k=1}^{n} \varphi_{\theta \delta L_{0}}\left(x_{k}\right)\right), \varphi_{\theta \delta L_{0}}\left(x_{n+1}\right)\right]=s^{-2} \sum_{k=1}^{n} i \Delta\left(x_{k}, x_{n+1}\right) W\left(\times_{j \neq k} \varphi_{\theta \delta L_{0}}\left(x_{j}\right)\right) .
$$

Since the time-ordered-products $T_{\theta \delta L_{0}}\left(\prod \Phi_{i}\left(f_{i}\right)\right)$ satisfy similar commutation relations with the field $\varphi_{\theta \delta L_{0}}(f)$ (see eq. (85)), it is possible to prove that, within $V$, these timeordered-products can expanded in terms of the products $W\left(\times_{i} \varphi_{\theta \delta L_{0}}\left(x_{i}\right)\right)$ in a manner analogous to the usual Wick expansion,

$$
\begin{aligned}
& T_{\theta \delta L_{0}}\left(\prod_{i=1}^{n} \varphi^{k_{i}}\left(x_{i}\right)\right)= \sum_{j \leq k}\left(\begin{array}{c}
k \\
j
\end{array}\right) \tau_{k_{1}-j_{1} \ldots k_{n}-j_{n}}\left(x_{1}, \ldots, x_{n}\right) \times \\
& W(\underbrace{\varphi_{\theta \delta L_{0}}\left(x_{1}\right), \ldots, \varphi_{\theta \delta L_{0}}\left(x_{1}\right)}_{j_{1} \text { times }}, \ldots, \underbrace{\varphi_{\theta \delta L_{0}}\left(x_{n}\right), \ldots, \varphi_{\theta \delta L_{0}}\left(x_{n}\right)}_{j_{n} \text { times }}),
\end{aligned}
$$

where the coefficients $\tau_{k_{1}-j_{1} \ldots k_{n}-j_{n}}$ are distributional and we use a multi-index notation $j=\left(j_{1}, \ldots, j_{n}\right), j !=\prod j_{i}$ ! , etc. The proof of this statement is similar to the proof of the Wick expansion for the time-ordered-products in the free field theory given in [15]. Namely, we assume inductively that eq. (98) has been demonstrated for all multi indices $k$ with $|k|=\sum k_{i}<m$. In order to prove it for a multi index $k$ with $|k|=m$, we consider the expression

$$
\begin{aligned}
D_{\theta}\left(x_{1}, \ldots, x_{n}\right)=T_{\theta \delta L_{0}}\left(\prod_{i=1}^{n} \varphi^{k_{i}}\left(x_{i}\right)\right)-\sum_{0 \neq j \leq k}\left(\begin{array}{c}
k \\
j
\end{array}\right) \tau_{k_{1}-j_{1} \ldots k_{n}-j_{n}}\left(x_{1}, \ldots, x_{n}\right) \times \\
W(\underbrace{\varphi_{\theta \delta L_{0}}\left(x_{1}\right), \ldots, \varphi_{\theta \delta L_{0}}\left(x_{1}\right)}_{j_{1} \text { times }}, \ldots, \underbrace{\varphi_{\theta \delta L_{0}}\left(x_{n}\right), \ldots, \varphi_{\theta \delta L_{0}}\left(x_{n}\right)}_{j_{n} \text { times }}),
\end{aligned}
$$

where the only term $\tau_{k_{1} \ldots k_{n}}$ that is not yet known by the induction hypothesis has been omitted from the sum in (99). The commutation relations for the individual terms on the right side of this equation now imply the commutation relation $\left[D_{\theta}\left(x_{1}, \ldots, x_{n}\right), \varphi_{\theta \delta L_{0}}(y)\right]=$ 0 within $V$. The above statements will still be true for a suitable $V$ containing a neighborhood of some Cauchy surface $\Sigma$ of $M$. In this case, one can easily prove using eq. (86) and the above commutation relation that $D_{\theta}$ must in fact be a multiple of the identity. We define $\tau_{k_{1} \ldots k_{n}}$ to be this multiple.

The products on the right side of eq. (98) can be written in terms of ordinary products using the formula

$$
W\left(\times_{i=1}^{N} \varphi_{\theta \delta L_{0}}\left(x_{i}\right)\right)=\sum_{P} \prod_{j \notin I \forall I \in P} \varphi_{\theta \delta L_{0}}\left(x_{j}\right) \prod_{P \ni I=\left\{i_{1}, i_{2}\right\}} \omega_{\theta \delta L_{0}}\left(x_{i_{1}}, x_{i_{2}}\right) .
$$


where $P$ runs over all sets of mutually disjoint subsets $I=\left\{i_{1}, i_{2}\right\}$ of $\{1, \ldots, N\}$ with 2 elements and where $\omega_{\theta \delta L_{0}}\left(x_{1}, x_{2}\right)=\omega\left(\varphi_{\theta \delta L_{0}}\left(x_{1}\right) \varphi_{\theta \delta L_{0}}\left(x_{2}\right)\right)$. Thus, since we already know that $s \varphi_{\theta \delta L_{0}}(x)$ is the image of $\varphi(x)$ under $\rho_{\theta}$, we get from formula (100) an algebraic element whose image under $\rho_{\theta}$ is $W\left(\times_{i} \varphi_{\theta \delta L_{0}}\left(x_{i}\right)\right)$. Once we have found those elements, we then get via eq. (98) algebraic elements $F\left(s ; \times_{i} f_{i} \Phi_{i}\right)$ in $\mathcal{X}(V, \mathbf{g})$ whose image under $\rho_{\theta}$ is $s^{N} T_{\theta \delta L_{0}}\left(\prod \Phi_{i}\left(f_{i}\right)\right)$.

It can be shown explicitly that the quantities $F\left(s ; \times_{i} f_{i} \Phi_{i}\right)$ are (s-dependent) local and covariant fields in the sense of our definition of local and covariant fields in the free theory (see eq. (10)), and that they have a smooth/analytic dependence on the metric under smooth/analytic variations of the metric. It is straightforward to show that the quantities $F\left(s ; \times_{i} f_{i} \Phi_{i}\right)$ satisfy the causal factorization property

$$
F\left(s ; \times_{i=1}^{n} f_{i} \Phi_{i}\right)=F\left(s ; \times_{i \in I} f_{i} \Phi_{i}\right) \star F\left(s ; \times_{j \in J} f_{j} \Phi_{j}\right)
$$

whenever $J^{-}\left(\operatorname{supp} f_{i}\right) \cap \operatorname{supp} f_{j}=\emptyset$ for all $(i, j) \in I \times J$, where $I \cup J=\{1, \ldots, n\}$ is a partition into disjoint sets. It can be shown from eq. (85) that the fields $F\left(s ; \times_{i} f_{i} \Phi_{i}\right)$ also satisfy the commutator property with a free field. Thus, these fields give a prescription $\tilde{T}\left(\prod \tilde{\Phi}_{i}\left(f_{i}\right)\right)$ for defining time-ordered-products to which our uniqueness theorem described in section 2 can be applied 21 .

By this uniqueness result, the relation between the prescription $\tilde{T}$ and the original prescription $T$ for time-ordered-products in the free theory is given by eq. (16). This is equivalent to

$$
\rho_{\theta}\left[S\left(\sum f_{i} \Phi_{i}\right)\right]=\mathcal{S}_{\theta \delta L_{0}}\left(s^{M_{i}} \sum f_{i} \Phi_{i}+\delta\left(s ; \sum f_{i} \Phi_{i}\right)\right)
$$

where the $\delta$ was introduced in eq. (17), and where $M_{i}$ is the number of factors of $\varphi$ in the field $\Phi_{i}$. (Note that $\delta$ now has an additional $s$-dependence, due to the fact that the prescription $\tilde{T}$ is $s$-dependent.) Equation (102) is the key identity for this proof. In order to exploit it, we introduce a cutoff function $\theta^{\prime}$ which equals 1 on $V$ and which is such that the support of $\theta^{\prime}$ is contained in the region where $\theta$ equals 1 . If we now apply $\rho_{\theta}$ to the element $\mathcal{S}_{\theta^{\prime} L_{1}}\left(\sum f_{i} \Phi_{i}\right)$, use eq. (102) and proceed in a similar way as in the proof of eq. (52) in section 3.2 to bring the resulting expression into a convenient form, then we obtain the identity

$$
\operatorname{Ad}\left(V\left(\theta, \theta^{\prime}\right)\right) \circ \rho_{\theta}\left[\mathcal{S}_{\theta^{\prime} L_{1}}\left(\sum f_{i} \Phi_{i}\right)\right]=\mathcal{S}_{\theta^{\prime} K_{1}(s)}\left(\sum N_{i}(s) f_{i} \Phi_{i}+\boldsymbol{\delta}_{L_{1}}\left(s ; \sum f_{i} \Phi_{i}\right)\right)
$$

for all test densities $f_{i}$ with support in $V$. Here, $V\left(\theta, \theta^{\prime}\right)$ is a unitary that is defined in a similar way as the unitary $W(\theta)$ in the proof of eq. (52) in section $3.2, N_{i}(s)$ are formal

\footnotetext{
21 Note however that the time-ordered-products $\tilde{T}\left(\prod \tilde{\Phi}_{i}\right)$ are by construction only defined as formal power series in $\mathcal{X}(V, \mathbf{g})$ rather than $\mathcal{W}(V, \mathbf{g})$, since they may depend on $s$ which is itself a formal power series in $\kappa$. It is however not difficult to see that our uniqueness theorem can nevertheless still be applied.
} 
power series in $s, \boldsymbol{\delta}_{L_{1}}$ is defined as in eq. (55), and $\boldsymbol{K}_{1}(s)$ is the interaction Lagrangian given by

$$
\boldsymbol{K}_{1}(s)=\left(s^{2}-1\right) \boldsymbol{L}_{0}+s^{4} \boldsymbol{L}_{1}+\left.\delta\left(s ; \theta \boldsymbol{L}_{1}\right)\right|_{\theta=1} .
$$

Finally, the desired *-isomorphism $\rho_{s}$ is then obtained from eq. (103) by removing the cutoff represented by $\theta$ and $\theta^{\prime}$ in the same way as in our construction of the interacting field given in section 3.1. Equation (81) corresponds to the special case $\Phi=\varphi$ of eq. (103).

We finally remark that, as indicated above, if the prescription $\tilde{T}$ given by eq. (93) were actually equal to the original prescription $T$ for defining the time-ordered-products, then the term $\delta\left(s ; \sum f_{i} \Phi_{i}\right)$ appearing in eq. (102) would be zero. This would imply that the factors $N_{i}(s)$ in eq. (103) is equal to $s^{M_{i}}$ (where $M_{i}$ is the number of factors of $\varphi$ in the field $\left.\Phi_{i}\right)$, the term $\boldsymbol{\delta}_{L_{1}}\left(s ; \sum f_{i} \Phi_{i}\right)$ in eq. (103) would vanish, and the Lagrangian $\boldsymbol{K}_{1}(s)$ would be equal to $\boldsymbol{L}_{1}(s)$ given by eq. (80) as in the classical theory. Thus, eq. (81) in the statement of the theorem would be simplified to $\rho_{s}\left[\varphi_{L_{1}}(f)\right]=s \varphi_{L_{1}(s)}(f)$, in complete analogy with the classical theory.

\section{B How to calculate the renormalization group in terms of Feynman diagrams}

In the previous sections we have set up a general framework for describing how a given perturbative interacting field theory in curved spacetime changes under a change of lengthscale, or, more properly, under a rescaling of the metric. This has led us to a completely satisfactory notion of the renormalization group flow in curved spacetime, without thereby having to introduce arbitrary vacuum states, bare couplings, cutoffs or arbitrary mass scales into the theory.

However, our construction is rather abstract and it may not be obvious how one would calculate this flow in practice (to a given order in perturbation theory). We will now outline how this can be done, and we will thereby establish the connection between the framework explained above and the formalism of Feynman diagrams, which is commonly used to define the renormalization group flow in Minkowski spacetime ${ }^{22}$.

To begin, we define [14, 15], for sufficiently nearby points, "locally normal ordered" fields : $\prod \varphi^{k_{i}}\left(x_{i}\right):_{H}$ by

$$
: \prod_{i=1}^{n} \varphi^{k_{i}}\left(x_{i}\right):_{H} \equiv \frac{\delta^{|k|}}{i^{|k|} \delta f\left(x_{1}\right)^{k_{1}} \ldots \delta f\left(x_{n}\right)^{k_{n}}} \exp \left[i \varphi(f)+\frac{1}{2} H(f, f)\right],
$$

where $|k|=\sum k_{i}$ and where

$$
H\left(x_{1}, x_{2}\right)=U\left(x_{1}, x_{2}\right) P\left(\sigma^{-1}\right)+V\left(x_{1}, x_{2}\right) \log |\sigma|
$$

\footnotetext{
${ }^{22}$ We have already noted at the end of section 4.1 that the functions $G_{1}, G_{3}, G_{4}$ appearing in the renormalization group flow (see eq. (78)) can be determined in Minkowski spacetime, and they can be calculated by standard methods. However, the function $G_{2}$ must be calculated in curved spacetime.
} 
is the "local Hadamard parametrix". Since $: \varphi^{k}(x):_{H}$ itself is a prescription for defining Wick powers to which our uniqueness theorem applies [14, it is possible to expand the Wick powers $\varphi^{k}(x)$ in a "local Wick expansion" in terms of these locally normal ordered fields [14],

$$
\varphi^{k}(x)=\sum_{j \leq k}\left(\begin{array}{l}
k \\
j
\end{array}\right) t_{k-j}(x): \varphi^{j}(x):_{H}
$$

where $t_{k}$ are finite sums of terms of the form local curvature terms times parameters in the free theory, of the appropriate engineering dimension. Of course, if the prescription for defining Wick powers is chosen to be that of "local normal ordering" with respect to $H$, then the expansion of eq. (107) is trivial, i.e., we have $t_{0}=1$ and $t_{j}=0$ for all $j>0$. A similar expansion is possible also for the time-ordered-products [15,

$$
T\left(\prod_{i=1}^{n} \varphi^{k_{i}}\left(x_{i}\right)\right)=\sum_{j \leq k}\left(\begin{array}{c}
k \\
j
\end{array}\right) t_{k_{1}-j_{1} \ldots k_{n}-j_{n}}\left(x_{1}, \ldots, x_{n}\right): \prod_{i=1}^{n} \varphi^{j_{i}}\left(x_{i}\right):_{H},
$$

where the $t_{j_{1} \ldots j_{n}}$ are certain distributions that are defined locally and covariantly in terms of the metric 5 , and where in eq. (109) we use the multi-index notation $j=\left(j_{1}, \ldots, j_{n}\right)$, $j !=\prod_{i} j_{i}$ ! etc.

The local Hadamard parametrices $H$ appearing in eqs. (107) and (108) could be chosen so that in Minkowski spacetime it coincides with the symmetrized two-point function of the unique, Poincare invariant vacuum state. In that case, when restricted to Minkowski spacetime, the "local normal ordering" prescription for defining Wick powers would coincide with the (globally defined) normal ordering with respect to the Poincare invariant vacuum state. Thus, in Minkowski spacetime, the expansion (109) could be viewed as expressing time-ordered-products in terms of normal ordered products with repect to the usual vacuum state. In curved spacetime, it also would be possible to choose a globally defined "vacuum state" (i.e., a quasi-free Hadamard state), $\omega$, and perform Wick expansions in terms of Wick products that are normal ordered with respect to $\omega$. This would have the advantage that the resulting coeficients $t$ would be globally defined rather than being defined only on a neighborhood of the total diagonal. However, it would have the major disadvantages that (i) the expansion (98) would always be nontrivial (since a local, covariant field cannot coincide with a normal ordered field on all spacetimes [14]) and (ii) the $t$ would no longer be locally and covariantly constructed out of the metric, so one could not evaluate the $t$ by local computations.

The distributions $t$ can further be decomposed into contributions from individual Feynman diagrams as follows. Let $\mathcal{F}^{(k)}$ be the set of all Feynman diagrams consisting with $n$ vertices located at the points $x_{1}, \ldots, x_{n}$ that are connected by a single kind of

\footnotetext{
${ }^{23}$ However, it should be noted that $t_{j_{1} \ldots j_{n}}$ is not actually a local, covariant (c-number) field in the sense of [5], since one cannot give a local, covariant prescription for how to choose the convex normal neighborhood that enters the definition of $H$.
} 
line, with the properties that the lines may emerge and end on two different vertices or they may emerge and end on the same vertex, and the $i$ th vertex has precisely $k_{i}$ edges emerging/ending on it. If $\Gamma$ is such a Feynman graph, then we denote by $E(\Gamma)$ the set of edges and by $V(\Gamma)$ the set of vertices. If $e$ is an edge, then we write $s(e)$ for the source of $e$ and $t(e)$ for its target. If $v$ is a vertex, then we write $n(v)$ for twice the number of edges that have $v$ both as their starting and endpoint. For points $x_{1}, \ldots, x_{n}$ such that $x_{i} \neq x_{j}$ for all $i, j$, we then have

$$
\begin{aligned}
t_{k_{1} \ldots k_{n}}\left(x_{1}, \ldots, x_{n}\right) & =\sum_{\Gamma \in \mathcal{F}^{(k)}} c^{\Gamma} \prod_{e \in E(\Gamma)} H_{F}\left(x_{s(e)}, x_{t(e)}\right) \prod_{v \in V(\Gamma)} t_{n(v)}\left(x_{v}\right) \\
& \equiv \sum_{\Gamma \in \mathcal{F}^{(k)}} t^{\Gamma}\left(x_{1}, \ldots, x_{n}\right)
\end{aligned}
$$

where $c^{\Gamma}$ are combinatorical factors and $H_{F}$ is the "local Feynman parametrix" given by

$$
H_{F}\left(x_{1}, x_{2}\right)=U\left(x_{1}, x_{2}\right)(\sigma+i 0)^{-1}+V\left(x_{1}, x_{2}\right) \log (\sigma+i 0) .
$$

Equation (109) can be viewed as giving the "Feynman rules" in curved spacetime. Mainly for simplicity, we have only considered explicitly only time-ordered-products of Wick powers without derivatives. Our discussion can be generalized to give similar Feynman rules also for time-ordered-products containing derivatives.

The Feynman rules in curved spacetime are thus very similar to those in Minkowski spacetime, with the local Feynman parametrix (100) replacing the usual Feynman propagator. However, there is one key difference in that if the prescription used for defining Wick powers does not coincide with "local normal ordering", then the Wick expansion (98) will be nontrivial, and there will be correspondingly nontrivial Feynman diagrams containing lines that begin and end at the same vertex.

The distributions $t^{\Gamma}$ in eq. (109) are locally and covariantly constructed from the metric and the coupling parameters in the free theory. They describe the contribution of an individual Feynman graph to a time-ordered-product. Formula (109) only determines them as distributions on the product manifold $M^{\times n}$ minus the union of all of its partial diagonals. A prescription for the extension of all time-ordered-products to all of $M^{\times n}$ is usually called "renormalization". The existence of a renormalization prescription satisfying a list of necessary properties was proven in [15] without going through the intermediate step of expanding the $t_{k_{1} \ldots k_{n}}$ in terms of Feynman diagrams.

Given the distributions $t^{\Gamma}$ corresponding to a given prescription $T$ for defining time ordered products, we can now obtain the corresponding rescaled prescription ${ }^{\lambda} T$ (see eq. (70)) as follows: If $p=\left(m^{2}, \xi\right)$ and $p(\lambda)=\left(\lambda^{-2} m^{2}, \xi\right)$, we first set

$$
t_{\lambda}^{\Gamma}[M, \mathbf{g}, p] \equiv \lambda^{2|E(\Gamma)|} \cdot t^{\Gamma}\left[M, \lambda^{2} \mathbf{g}, p(\lambda)\right]
$$

as well as

$$
H_{\lambda}[M, \mathbf{g}, p] \equiv \lambda^{2} \cdot H\left[M, \lambda^{2} \mathbf{g}, p(\lambda)\right]
$$


The rescaled prescription ${ }^{\lambda} T$ is then given by

$$
{ }^{\lambda} T\left(\prod_{i=1}^{n}{ }^{\lambda} \varphi^{k_{i}}\left(x_{i}\right)\right)=\sum_{j \leq k} \sum_{\Gamma \in \mathcal{F}^{(k-j)}} t_{\lambda}^{\Gamma}\left(x_{1}, \ldots, x_{n}\right): \prod_{i=1}^{n} \varphi^{j_{i}}\left(x_{i}\right):_{H_{\lambda}} .
$$

Given the rescaled prescription ${ }^{\lambda} T$, we can now compute the maps $O_{n}\left(\lambda ; \times_{i} f_{i} \Phi_{i}\right)$ (see eq. (30), which relate the rescaled prescription to the original prescription $T$. The renormalization group flow $\boldsymbol{L}_{1}(\lambda)$ is then given in terms of these quantities by given by

$$
\delta \boldsymbol{L}_{1}(\lambda)=\left.\sum_{n=1}^{\infty} \frac{i^{n-1}}{n !} O_{n}\left(\lambda ; \times{ }^{n} \theta \boldsymbol{L}_{1}\right)\right|_{\theta=1} .
$$

Each term in the sum (113) is of the form (51) for some real coupling constants $\delta m^{2(n)}$, $\delta z^{(n)}, \delta \xi^{(n)}$, and $\delta \kappa^{(n)}$, each of which is a polynomial in $\log \lambda$. These quantities are the renormalization group flow at $n$-th order in perturbation theory.

This completes our brief discussion on how to calculate the renormalization group flow in terms of Feynman diagrams. We note, however, that the calculation of the $\beta$-function as defined by (83) is more complicated since it also requires the calculation of $\boldsymbol{K}_{1}(s)$ (see appendix A).

\section{References}

[1] F. M. Boas: "Gauge theories in local causal perturbation theory," DESY-THESIS 1999-032, (1999) [arXiv: hep-th/0001014]

[2] N. N. Boboliubov and D. V. Shirkov: "Introduction to the theory of quantized fields," New York (1959)

[3] R. Brunetti, K. Fredenhagen and M. Köhler: "The microlocal spectrum condition and Wick polynomials on curved spacetimes," Commun. Math. Phys. 180, 633-652 (1996)

[4] R. Brunetti and K. Fredenhagen: "Microlocal Analysis and Interacting Quantum Field Theories: Renormalization on physical backgrounds," Commun. Math. Phys. 208, 623-661 (2000)

[5] R. Brunetti, K. Fredenhagen and R. Verch, "The generally covariant locality principle: A new paradigm for local quantum physics," arXiv:math-ph/0112041.

[6] B. S. DeWitt and R. W. Brehme: "Radiation Damping In A Gravitational Field," Annals Phys. 9 (1960) 220 
[7] J. Dieckmann, "Cauchy surfaces in globally hyperbolic spacetimes," J. Math. Phys. 29, 578 (1988).

[8] M. Dütsch and K. Fredenhagen: "Algebraic quantum field theory, perturbation theory, and the loop expansion," Commun. Math. Phys. 219, 5 (2002) [arXiv: hepth/0001129]; "Perturbative algebraic field theory, and deformation quantization," [arXiv: hep-th/0101079]

[9] M. Dütsch and K. Fredenhagen: "A local (perturbative) construction of observables in gauge theories: The example of QED," Commun. Math. Phys. 203, 71 (1999) arXiv:hep-th/9807078

[10] R. Geroch: "Domain of Dependence," J. Math. Phys. 11, 437 (1970)

[11] V. Glaser, H. Lehmann, W. Zimmermann: "Field operators and retarded products," Nuovo Cim. 6, 1122 (1957)

[12] R. Haag: "On quantum field theories," Dan. Mat. Fys. Medd. 29, 13 (1955) no. 12, reprinted in: Dispersion Relations and the Abstract Approach to Field Theory, L. Klein (ed.), Gordon \& Breach, NY, 1961.

[13] L. Hörmander: "The Analysis of Linear Partial Differential Operators I," SpringerVerlag, Berlin 1985

[14] S. Hollands and R. M. Wald: "Local Wick Polynomials and Time-Ordered-Products of Quantum Fields in Curved Space," Commun. Math. Phys. 223, 289-326 (2001)

[15] S. Hollands and R. M. Wald: "Existence of local covariant time-ordered-products of quantum fields in curved spacetime," Commun. Math. Phys. (in press) arXiv:grqc/0111108

[16] B. S. Kay and R. M. Wald: "Theorems on the uniqueness and thermal properties of stationary, nonsingular, quasifree states on spacetimes with a bifurcate Killing horizon," Phys. Rep. 207, 49 (1991)

[17] V. Moretti: "Comments on the stress-energy tensor operator in curved spacetime," arXiv:gr-qc/0109048

[18] B. L. Nelson and P. Panangaden: "Scaling behavior of interacting quantum fields in curved spacetime," Phys. Rev. D 25, 1019-1027 (1982)

[19] B. L. Nelson and P. Panangaden: "Universality and quantum gravity," Phys. Rev. D 29, 2759-2762 (1984) 
[20] G. Pinter: "Finite Renormalizations in the Epstein Glaser Framework and renormalization of the S-matrix in $\varphi^{4}$-theory," [arXiv: hep-the/9911063]

[21] L. Parker and D. J. Toms: "Renormalization-group analysis of grand unified theories in curved spacetime," Phys. Rev. D29 1584-1604 (1984)

[22] R. M. Wald: "Quantum Field Theory on Curved Spacetimes and Black Hole Thermodynamics," The University of Chicago Press, Chicago (1990)

[23] S. Weinberg: "Ultraviolet divergences in quantum theories of gravitation," in: General Relativity, eds. S. W. Hawking and W. Israel, Cambridge University Press (1979) 